\title{
Análisis psicométrico del Inventario de Estrategias de Aprendizaje y Estudio en estudiantes universitarios de psicología de Lima metropolitana*
}

\author{
Luis Miguel Escurra Mayaute
}

Se realizó una investigación de tipo metodológico de la Validez y Confiabilidad del Inventario de Estrategias de Aprendizaje y Estudio (IEAE), también conocido como LASSI, por sus siglas en inglés. El diseño aplicado correspondió al descriptivo simple y fue efectuado en una muestra de 566 estudiantes universitarios de psicología de Lima metropolitana. El análisis comparativo indicó que los alumnos que estudian en universidades particulares presentaron un mejor desempeño en las escalas de Actitud, Motivación y Autoevaluación. También se apreció que existieron diferencias por sexo, pues las mujeres presentaron un mejor desempeño que los varones en las escalas de Actitud, Administración del Tiempo, Ansiedad y Ayudas.

Estrategias de estudio y aprendizaje / Validez / Confiabilidad / estudiantes de psicología

Psychometric analysis of the Inventory of Learning and Study Strategies Inventory (LASSI) in psychology university students in metropolitan Lima

This is a methodological investigation of the validity and reliability of the Inventory of Learning and Study Strategies Inventory (LASSI). The simple descriptive design was applied to 566 university students of psychology in metropolitan Lima, using a multi-phased stratified sample. The comparative analysis indicated that students of private universities showed better performance than students from public universities in the scales of Attitude, Motivation and Self-Testing. Differences by sex in the scales of Attitude, Time Management, Anxiety and Study Aids were observed.

Learning and study strategies / Validity / Reliability / Psychology students

* Estudio auspiciado por el Instituto de Investigación Científica de la Universidad de Lima. 


\section{INTRODUCCIÓN}

En los últimos años el interés por la calidad de la enseñanza universitaria se ha incrementado de manera sustancial. Hasta hace poco tiempo la mayoría de los trabajos empíricos sobre el aprendizaje autorregulado se habían centrado en el estudio de los escolares, pero la enseñanza en la escuela tradicional se ha caracterizado por la presencia de un fuerte control externo, en el cual el maestro asume la responsabilidad de orientar y regular el aprendizaje de sus alumnos (Vermunt, 1995), limitando de esta manera la investigación sobre la autorregulación del aprendizaje.

La experiencia universitaria es una etapa crítica en el desarrollo estudiantil, debido a que los futuros profesionales se preparan para tomar las decisiones importantes en la sociedad del futuro. El proceso interactivo de enseñanza aprendizaje comienza en la sala de clases, lugar donde surgen las diferentes actividades básicas para el proceso de transformación de los estudiantes y de los profesores. Las actividades, que son el elemento fundamental del proceso de aprendizaje, demuestran una variación amplia entre los patrones, los estilos y la calidad de lo que se enseña.

Mayor, Suengas y González (1993) y Beltrán (1996) reportan la importancia de estudiar la relación de lo que se enseña y cómo lo aprenden los estudiantes, es decir el contenido en contraposición con el proceso. De esta manera resulta importante identificar las estrategias de aprendizaje en jóvenes y adultos que predominan en el nivel universitario (Quesada, 2001; Zabalza, 2002, 2003).

En el contexto del aprendizaje universitario se proporcionan mejores condiciones para la investigación de la autorregulación, porque el control del profesor es menor y se espera que sea el propio estudiante quien controle sus intenciones de aprendizaje, se imponga sus propias metas, distribuya su tiempo y esfuerzo y mantenga su motivación.

Los objetivos de las enseñanzas superiores asumen, tal como en otros niveles educativos, la importancia de la autorregulación en el estudio de los alumnos universitarios como una de las condiciones para un aprendizaje de calidad, como resaltan las teorías actuales del aprendizaje.

El éxito y el fracaso académicos en el ámbito universitario es un tema que adquiere mayor importancia en la investigación educativa moderna, y sobre el que existe acuerdo en cuanto a ubicar su origen en la confluencia de múltiples factores, entre los que destacan el uso de inadecuadas estrategias de aprendizaje, por lo cual se hace indispensable contar con instrumentos válidos y confiables que permitan evaluar estos aspectos relacionados con el aprendizaje cognitivo.

Es a partir de estas consideraciones que en el presente estudio se planteó la necesidad de adaptar el Inventario de 
Estrategias de Aprendizaje y Estudio (IEAE), en los estudiantes universitarios de psicología de Lima metropolitana, de manera que la información obtenida permita conocer las características de las estrategias de aprendizaje que presentan los alumnos de psicología en diferentes tipos de universidades de Lima metropolitana, facilitando la identificación de las fortalezas y debilidades que estos presentan en dichos aspectos.

Adicionalmente, la investigación se propuso averiguar cuáles son las estrategias de aprendizaje predominantes entre nuestros estudiantes, de manera que brinden información valiosa para tratar de optimizar los procesos de aprendizaje autorregulado de los alumnos y les permitan realmente aprender a aprender.

Es necesario resaltar que la adaptación de pruebas que actualmente se administran en otros contextos nos va a permitir contar con instrumentos adecuados a nuestra realidad educativa y que tengan validez y confiabilidad en las intervenciones profesionales que pudieran realizarse en nuestro medio. Esto es algo que debe remarcarse, pues como se sabe, una de las grandes carencias en la investigación educativa y psicológica en nuestro medio, es, precisamente, la falta de instrumentos que sean válidos, confiables y adaptados a nuestra realidad cultural.

\section{CONCEPTOS BÁSICOS}

En todos los niveles educativos nos encontramos con numerosos problemas de aprendizaje, sabemos que son muchos los factores que pueden influir en un momento determinado, sin embargo, existe evidencia de que uno de estos factores es no saber cómo aprender; esto implica que la mayoría de los estudiantes no utilizan las estrategias adecuadas para lograr un aprendizaje significativo (Nisbet \& Schucksmith, 1986).

Desde hace poco tiempo se ha puesto más atención en lo que se ha llamado "aprender a aprender", es decir, enseñar al alumno a emplear las estrategias de aprendizaje más adecuadas en la adquisición de nuevos conocimientos, es por ello que se revisarán los principales conceptos relacionados con este tema.

Algunos de los aspectos más importantes de la educación son las nociones sobre intenciones, planes y esfuerzos que realiza el alumno; sin embargo, estos aspectos no han recibido la atención debida y han tendido a caer en el vacío que hay entre las corrientes de investigación conductual y la corriente constructivista.

La ciencia cognoscitiva es una ciencia formada por las aportaciones derivadas de disciplinas y concepciones teóricas diversas, como la teoría de Piaget (1975), la psicolingüística y la teoría de la información. Recientemen- 
te, la ciencia cognoscitiva contemporánea ha empezado a penetrar en las dos corrientes arriba mencionadas, con la intención de acercarlas; y lo que las acerca es, precisamente, lo que se ha llamado aprendizaje intencional.

El aprendizaje intencional se refiere a los procesos cognoscitivos que tienen como meta lograr un determinado aprendizaje en lugar de un resultado incidental (Bereiter \& Scardamalia, 1989). Este aprendizaje depende tanto de los factores situacionales externos como de los factores internos, es decir que este término coordina de forma natural la tradición que trata con las situaciones de aprendizaje y la tradición que se orienta sobre las habilidades de aprendizaje.

Lo anterior permite aclarar que este tipo de aprendizaje puede ocurrir en una situación dirigida por el maestro o bien en una situación autodirigida por el alumno.

La mayoría de las investigaciones sobre el aprendizaje intencional se encuentran bajo el nombre de habilidades de estudio o estrategias de aprendizaje. Estas investigaciones han tratado principalmente sobre procedimientos de autorregulación, en donde se encuentra implícito el repertorio estratégico del aprendizaje intencional, dos conclusiones importantes derivadas de estas investigaciones son las siguientes:

- Existe una gran variedad de estrategias para lograr el aprendizaje signi- ficativo que muchos estudiantes no aplican, y los programas de entrenamiento sobre estrategias de aprendizaje han demostrado ser efectivos e incluso generalizados en la mayoría de las ocasiones, como lo demuestran Palincsar y Brown (1984).

- Los métodos de enseñanza son los responsables de las estrategias utilizadas por los estudiantes. Como indica Moreno (1989), la forma en que los profesores presentan el conocimiento, la cantidad y tipo de información que se ofrece, las preguntas que se plantean y el método de evaluación favorecen el desarrollo del metaconocimiento y ciertas estrategias de aprendizaje más adecuadas o todo lo contrario.

La mayoría de las veces la escuela fomenta que los estudiantes utilicen solo aquellas estrategias que permiten alcanzar metas a corto plazo, y muy pocas veces aprenden a integrar la información o a construir un conocimiento con un valor a largo plazo (Bereiter \& Scardamalia, 1989). Con frecuencia los métodos de enseñanza empleados en las escuelas no solo no fomentan el uso de estrategias de aprendizaje adecuadas, sino que en muchos casos impiden y bloquean su desarrollo.

\section{LAS ESTRATEGIAS DE APRENDIZAJE Y LAS HABILIDADES METACOGNITIVAS}

Existen distintas definiciones sobre estrategias de aprendizaje, entre las que 
destacan las propuestas por los siguientes autores:

- Presley, Presley, Elliot-Faust \& Miller (1985), los cuales mencionan que una estrategia está compuesta de operaciones cognoscitivas además de los procesos que son la consecuencia natural de llevar a cabo la tarea, abarcando desde una operación determinada a una secuencia de operaciones interdependientes. Las estrategias alcanzan propósitos cognitivos, como la comprensión y la memorización, y son actividades potencialmente controlables y conscientes.

- Derry \& Murphy (1986) indican que las estrategias de aprendizaje son un conjunto de conocimientos y procesos mentales empleado por una persona en una situación particular de aprendizaje para facilitar la adquisición de conocimientos.

- Weinstein y Mayer (1986), por su parte, plantean que las estrategias de aprendizaje pueden ser definidas como conductas y pensamientos que se emplean durante el aprendizaje y que intentan influir en los procesos de codificación del aprendiz. Así, la meta de cualquier estrategia de aprendizaje particular puede afectar los estados motivacionales y afectivos del aprendiz, o la forma en la que este selecciona, adquiere, organiza o integra el nuevo conocimiento.
- Tanto Dansereau (1985) como Nisbet y Shucksmith (1986), definen las estrategias como secuencias integradas de procedimientos o actividades que se eligen con el propósito de facilitar la adquisición, almacenamiento y/o utilización de la información.

Si se toman en cuenta todas estas definiciones, se puede decir que las estrategias de aprendizaje son actividades físicas (conductas, operaciones) y/o mentales (pensamientos, procesos cognoscitivos) que se llevan a cabo con un propósito cognoscitivo determinado, como sería el mejorar el aprendizaje, resolver un problema o facilitar la asimilación de la información. En lo que respecta a los estados afectivos y motivacionales, estos son fundamentales, si no hay un interés por el aprendizaje, este simplemente no se dará.

En lo que corresponde a los aspectos metacognitivos, respecto a su inclusión o no como un componente de las estrategias de aprendizaje. Si se analiza bajo el afronte piagetano de la toma de conciencia se puede decir que no. Pero la respuesta sería positiva si se acepta que un aspecto distintivo de la metacognición es la toma de conciencia de las estrategias empleadas; cuando estas son inconscientes se encuentran inmersas solo en esquemas de acción, lo que no permite reflexionar sobre ellas, pero esto es posible cuando las estrategias son conscientes, porque han pasado al plano de la conceptualización, es decir 
que están integradas a los esquemas conceptuales que posee el sujeto.

Moreno (1988) nos dice, refiriéndose a Piaget, que el paso de lo inconsciente a lo consciente significa una reconstrucción en el plano de la conceptualización, una transformación de un esquema de acción en un concepto, la toma de conciencia no se limita a iluminar aspectos ya dados, sino que construye otros nuevos esquemas. Por ejemplo, las estrategias utilizadas inconscientemente para resolver un problema determinado se encuentran en el plano de la acción, se puede resolver el problema pero no se puede explicar cómo o qué estrategias se emplearon. Aquí está actuando el mecanismo de la represión cognitiva, el esquema de acción (empleo de estrategia) no está en la conceptualización consciente al ser incompatible con los esquemas conceptuales que se tienen. Para hacerlos conscientes es necesario reorganizar o construir nuevos esquemas, esto es reconstruir en el plano de la conceptualización. Por lo tanto, cuando se habla de metacognición se está en el plano de la conceptualización y en el de las abstracciones reflejadas, y esto es lo que permite reflexionar sobre lo que se ha hecho, sobre el conocimiento que se tiene, y por consiguiente, después se puede llevar a cabo la autorregulación, la regulación consciente. De manera que no podemos decir que la metacognición sea un componente de las estrategias porque lo que hace posible la metacognición son los esquemas conceptuales, a los cuales las estrategias se encuentran integradas.

Por otro lado, nos referimos a las estrategias de aprendizaje como cualquier comportamiento, pensamiento, creencia o emoción que ayude a adquirir información e integrarla al conocimiento ya existente, así como a recuperar la información disponible (Weinstein, 1987; Weinstein, Husman \& Dierking, 2000).

Las estrategias de aprendizaje pueden ser caracterizadas como procedimentales, en el sentido de que pueden definirse como procedimientos; intencionales, puesto que tienen un carácter deliberado; requieren esfuerzo; son voluntarias; esenciales, necesarias en los comportamientos de personas expertas en un área y facilitadoras, porque en general se ha encontrado que mejoran el desempeño académico (Rinaudo \& Vélez, 2000).

Las estrategias son diferentes de las destrezas. Duffy y Roehler (1989) señalan lo relevante de esta distinción cuando dicen que si los maestros piensan que van a enseñar destrezas, el trabajo académico que ellos asignan hace pensar a los alumnos que ellos tienen que memorizar algo para inmediatamente recordarlo, algo parecido a aplicar una receta de cocina, en lugar de desarrollar planes flexibles susceptibles de adaptarse a diferentes situaciones. Esto último es lo que se pretende con la instrucción de estrategias, que a 
diferencia de las destrezas involucran el desarrollo de la conciencia metacognitiva de las estrategias y las expectativas de que estas serán aplicadas eventualmente de manera automática.

Las estrategias, en contraste con las destrezas, tienen un carácter idiosincrático y no se aplican siempre de la misma manera, en cambio las destrezas son procedimientos uniformemente aplicados, es decir que se aplican siempre igual en todas las situaciones.

Con relación a la metacognición, se puede indicar que existen dificultades para definirlo. Una de las principales fuentes de confusión, según Brown (1987), radica en que la literatura psicológica moderna ha utilizado el término para referirse a dos áreas de investigación distintas, como son el conocimiento sobre la cognición y la regulación de la cognición. Además, afirma que estas dos formas de metacognición están en realidad íntimamente relacionadas, sin embargo se las debe diferenciar por tener raíces históricas distintas y problemas diferentes que atender.

Por un lado, el conocimiento sobre la cognición, el saber sobre el conocimiento, es aquella información que se tiene sobre los propios procesos cognoscitivos y contenidos de conocimiento; este tipo de información, indica Brown (1987), tiene las siguientes características: es estable, a veces falible, se desarrolla tardíamente y se puede constatar su ocurrencia, esto implica que es consciente, es accesible al suje- to y por lo tanto puede reflexionar sobre ella y discutirla con otros.

Por el contrario, la regulación de la cognición, es una actividad inestable y relativamente independiente de la edad; depende más bien de las situaciones y tareas de aprendizaje, y tampoco se puede constatar su ocurrencia siempre, a veces no está a nivel consciente, y por lo tanto ni puede expresarla a los demás ni mucho menos reflexionar sobre ella.

Para Taylor (1983), las habilidades metacognitivas se refieren al conocimiento individual acerca de la tarea, las posibles estrategias que pueden ser aplicadas a la tarea y la conciencia individual de sus propias habilidades en relación con estas estrategias. Si la habilidad metacognitiva puede ser conceptualizada como un aspecto general del desarrollo cognitivo, entonces la metacognición es mucho más que la suma de las habilidades y estrategias individuales. Probablemente podría describirse como una tendencia general o predisposición para analizar tanto las tareas como las respuestas y reflexionar sobre las consecuencias de las respuestas.

Es importante resaltar la toma de conciencia tanto de los propios procesos cognoscitivos y contenidos de conocimiento como de las actividades de regulación de la cognición, pues nos interesa que las actividades de regulación sean conscientes, producto de la reflexión. 


\section{Procesos COGNOSCITIVOS IMPLICADOS EN LAS ESTRATEGIAS DE APRENDIZAJE}

Weinstein y Mayer (1986) proporcionan un marco para analizar el proceso de enseñanza-aprendizaje de las estrategias, dicho marco está constituido por los siguientes elementos:

- Las características del maestro: lo que este sabe y cómo lo enseña.

- Las estrategias de enseñanza: lo que el maestro hace durante la enseñanza.

- Las características del alumno: lo que este sabe sobre los hechos, procedimientos y estrategias que se requieren.

- Estrategias de aprendizaje: incluye aquellas conductas que el alumno lleva a cabo durante el aprendizaje y que son realizadas para influir en los procesos cognoscitivos y afectivos durante la codificación.

- Procesos de codificación: considera aquellos procesos cognoscitivos internos que se dan durante el aprendizaje, tales como la selección, organización e integración de la nueva información.

- Resultado del aprendizaje: se refiere a lo que se aprendió.

- Ejecución: se refiere a la manera en que se evalúa el aprendizaje, por ejemplo a través de pruebas de retención y transferencia.

Todos estos elementos son necesarios para la enseñanza de las estrate- gias; sin embargo, los procesos de codificación constituirían la clave del éxito o el fracaso del aprendizaje.

Los procesos de codificación son aquellos procesos cognoscitivos que pueden ser analizados dentro de cuatro componentes principales:

- La selección.- El alumno presta atención a la información que se le presenta y la transfiere a la memoria operativa.

- La adquisición.- Se transfiere la información de la memoria operativa a la memoria a largo plazo, con la finalidad de almacenarla permanentemente.

- Construcción.- Consiste en relacionar las ideas que hay en la información que se encuentra en la memoria operativa. Es decir, el alumno construye conexiones internas para darle a la información recibida una organización coherente.

- Integración.- El alumno busca, en su memoria a largo plazo, el conocimiento previo para transferirlo a la memoria operativa, y entonces realiza conexiones externas entre la nueva información y el conocimiento previo.

Los procesos de selección y adquisición determinan cuánto se aprende (la cantidad), mientras que la construcción y la integración son procesos cognoscitivos que determinan la coherencia de la organización de lo que es 
aprendido y sobre cómo es organizado (Weinstein \& Mayer, 1986).

Lo importante al considerar estos procesos en la enseñanza de las estrategias de aprendizaje y habilidades metacognoscitivas es que si queremos que el alumno alcance un aprendizaje significativo, de tal manera que se dé la transferencia, es decir que sea capaz de aplicar lo aprendido a situaciones diferentes, se procurará que el estudiante pase por los cuatro procesos cognoscitivos. Por ejemplo, en una tarea de comprensión de lectura se tratará de proporcionar aquellas estrategias que le permitan, primero, seleccionar y adquirir información, como subrayar o extraer las ideas claves del texto, y, posteriormente, elaborar un resumen y utilizar las estrategias de elaboración e imaginación para construir e integrar la nueva información.

En lo que respecta a las habilidades metacognoscitivas, también sería de mucha utilidad que los propios estudiantes conocieran en qué consisten estos procesos cognoscitivos (considerando su nivel de desarrollo), ya que les proporcionaría esquemas conceptuales para reflexionar sobre su aprendizaje y poder llevar a cabo una autorregulación consciente.

Analizando la clasificación realizada por Flavell y Wellman (en Moreno, 1989) sobre las variables de la persona, de la tarea y las estrategias, se puede sugerir que para diseñar programas de entrenamiento en estrategias de apren- dizaje y habilidades metacognitivas, debemos tomar en cuenta por lo menos lo siguiente:

- Las características de la población a la cual va dirigido: edad, nivel de escolarización, capacidades y limitaciones cognitivas, tipo de estrategias que emplean, etcétera.

- Características de la tarea: el tipo (comprensión de lectura, cálculo, matemáticas, física, manualidades, idiomas), el grado de dificultad, la estructuración del material, etcétera.

- Las estrategias de aprendizaje (sin olvidar los procesos cognoscitivos implicados): repetición, elaboración, organización, regulación, etcétera.

Antes y después de llevar a cabo cualquier tipo de entrenamiento es necesario evaluar las estrategias de los alumnos, y con esto entramos al problemático tema de la evaluación de las estrategias cognitivas y metacognitivas.

\section{Clasificación de las estrategias de APRENDIZAJE EN EL ÁMBITO ACADÉMICO}

Se han identificado cinco clases de estrategias generales en el ámbito educativo: las tres primeras ayudan al alumno a elaborar y organizar los contenidos para que resulte más fácil el aprendizaje (procesar la información); la cuarta está destinada a controlar la actividad mental del alumno, para dirigir el 
aprendizaje; y, por último, la quinta sirve de apoyo al aprendizaje, para que este se produzca en las mejores condiciones posibles. Estas estrategias son las siguientes:

- Estrategias de ensayo.- Son aquellas que implican la repetición activa de los contenidos (diciendo, escribiendo), o centrarse en partes claves de él.

- Estrategias de elaboración.- Implican hacer conexiones entre lo nuevo y lo familiar.

- Estrategias de organización.-Agrupan la información para que sea más fácil recordarla. Implican imponer una estructura a los contenidos de aprendizaje, dividiéndolo en partes e identificando relaciones $\mathrm{y}$ jerarquías.

- Estrategias de control de la comprensión. Son las estrategias ligadas a la metacognición. Implican permanecer consciente de lo que se está tratando de lograr, seguir la pista de las estrategias que se usan y del éxito logrado con ellas, y adaptar la conducta en concordancia. Si utilizásemos la metáfora de comparar la mente con un ordenador, estas estrategias actuarían como un procesador central de ordenador. Son un sistema supervisor de la acción y el pensamiento del alumno, y se caracterizan por un alto nivel de conciencia y control voluntario. Entre las estrategias metacognitivas están:
- Estrategias de planificación, aquellas mediante las cuales los alumnos dirigen y controlan su conducta. Son, por tanto, anteriores a que los alumnos realicen ninguna acción.

- Estrategias de regulación, dirección y supervisión, se utilizan durante la ejecución de la tarea. Indican la capacidad que el alumno tiene para seguir el plan trazado y comprobar su eficacia.

- Estrategias de evaluación, son las encargadas de verificar el proceso de aprendizaje. Se 1levan a cabo durante y al final del proceso.

- Estrategias de apoyo o afectivas.No se dirigen directamente al aprendizaje de los contenidos. La misión fundamental de estas estrategias es elevar el nivel de la eficacia del aprendizaje, mejorando las condiciones en las que se produce.

Algunos autores relacionan las estrategias de aprendizaje con un tipo determinado de aprendizaje. Para estos autores cada tipo de aprendizaje (por asociación o por reestructuración) estaría vinculado a una serie de las estrategias que le son propias.

Con respecto al tema de los tipos de estrategias de aprendizaje, Weinstein y Mayer (1986) distinguen tres grandes grupos de estrategias:

- Las estrategias cognitivas, que aluden a la integración del nuevo ma- 
terial con el conocimiento previo. En este sentido, serían un conjunto de estrategias que se utilizan para aprender, codificar, comprender y recordar la información al servicio de unas determinadas metas de aprendizaje. Dentro de este grupo se distinguen, a su vez, tres clases de estrategias: la estrategia de repetición, que consiste en pronunciar, nombrar o decir de forma repetida los estímulos presentados dentro de una tarea de aprendizaje. Se trataría, por tanto, de un mecanismo de la memoria a corto plazo y a la vez, transferidos a la memoria a largo plazo. Por otro lado, mientras que la estrategia de elaboración trata de integrar los materiales informativos, relacionando la nueva información con la ya almacenada en la memoria, la estrategia de organización intenta combinar los elementos informativos seleccionados en un todo coherente y significativo. Además, dentro de esta categoría de estrategias cognitivas también estarían las estrategias de selección, cuya función principal es la de seleccionar aquella información más relevante con la finalidad de facilitar su procesamiento.

Las estrategias metacognitivas hacen referencia a la planificación, control y evaluación por parte de los estudiantes de su propia cognición. Son un conjunto de estrategias que permiten el conocimiento de los procesos mentales, así como el control y regulación de estos con el objetivo de lograr determinadas metas de aprendizaje. Según Kirby (1977), este tipo de estrategias serían macroestrategias, ya que son más complejas que las anteriores, presentan un elevado grado de transferencia, son menos susceptibles de ser enseñadas, y están estrechamente relacionadas con el conocimiento metacognitivo.

El conocimiento metacognitivo requiere conciencia y conocimiento de variables de la persona, de la tarea y de la estrategia. En relación con las variables personales está la conciencia y conocimiento que tiene el sujeto de sí mismo y de sus capacidades y limitaciones cognitivas; aspecto que se va formando a partir de las percepciones y comprensiones que desarrollamos nosotros mismos, en tanto sujetos que aprenden y piensan. Las variables de la tarea se refieren a la reflexión sobre el tipo de problema que se va a tratar de resolver. Significa, por tanto, averiguar el objetivo de la tarea. En este sentido, puede entenderse la conciencia (conocimiento) metacognitiva como un proceso de utilización de pensamiento reflexivo para desarrollar la conciencia y conocimiento sobre uno mismo, la tarea, y las estrategias en un contexto determinado. 
Por consiguiente, una buena base de conocimientos de las características y demandas de la tarea, de las capacidades, intereses y actitudes personales, y de las estrategias necesarias para completar la tarea, son requisitos básicos de la conciencia y conocimientos metacognitivos; a lo que debemos de añadir la regulación y control que el propio sujeto debe ejercer sobre todo lo anterior. Para Kurtz-Costes y Schneider (1994), la metacognición regula de dos formas el uso eficaz de las estrategias: en primer lugar, para que un individuo pueda poner en práctica una estrategia, antes debe tener conocimiento de estrategias específicas y saber cómo, cuándo y por qué debe usarlas. Así, por ejemplo, debe conocer las técnicas de repaso, subrayado, resumen, etcétera; y saber cuándo conviene utilizarlas. En segundo lugar, mediante su función autorreguladora, la metacognición hace posible observar la eficacia de las estrategias elegidas y cambiarlas según las demandas de la tarea.

Las estrategias metacognitivas equivalen a lo que Weinstein \& Mayer (1986) denominan como estrategias de control de la comprensión. Estas estrategias están formadas por procedimientos de autorregulación que hacen posible el acceso consciente a las habilidades cognitivas empleadas para procesar la infor- mación. Para estos autores, un estudiante que emplea estrategias de control es también un estudiante metacognitivo, ya que es capaz de regular el propio pensamiento en el proceso de aprendizaje.

- Las estrategias de manejo de recursos son una serie de estrategias de apoyo, que incluyen diferentes tipos de recursos que contribuyen a que la resolución de la tarea se lleve a buen término. Tienen como finalidad sensibilizar al estudiante con lo que va a aprender; y esta sensibilización hacia el aprendizaje integra tres ámbitos: la motivación, las actitudes y el afecto. Este tipo de estrategias coinciden con lo que Weinstein y Mayer (1986) llaman estrategias afectivas y otros autores denominan estrategias de apoyo, e incluyen aspectos claves que condicionan el aprendizaje, como son el control del tiempo, la organización del ambiente de estudio, el manejo y control del esfuerzo, etcétera. Este tipo de estrategias, en lugar de enfocarse directamente sobre el aprendizaje tendrían como finalidad mejorar las condiciones materiales y psicológicas en que se produce ese aprendizaje. Gran parte de las estrategias incluidas dentro de esta categoría tiene que ver con la disposición afectiva y motivacional del sujeto hacia el aprendizaje.

La importancia de los componentes afectivo-motivacionales en 
la conducta estratégica es puesta de manifiesto por la mayor parte de los autores que trabajan en este campo. Todos coinciden en manifestar que los motivos, intenciones y metas de los estudiantes determinan en gran medida las estrategias específicas que utilizan en tareas de aprendizaje particulares. Por eso se entiende que la motivación es un componente necesario de la conducta estratégica y un requisito previo para utilizar estrategias.

Todo esto indica que los estudiantes suelen disponer de una serie de estrategias para mejorar el aprendizaje, aunque la puesta en marcha de estas depende, entre otros factores, de las metas que persigue el alumno, referidas tanto al tipo de metas académicas (por ejemplo, metas de aprendizaje o metas de rendimiento) como a los propósitos e intenciones que guían su conducta ante una tarea de aprendizaje en particular.

Para Biggs (1993) el estudio de las estrategias de aprendizaje realizado por los investigadores se sustenta en dos afrontes teóricos totalmente diferentes:

- El modelo de procesamiento de la información, que orienta a establecer cuál es el significado que tienen las estrategias de aprendizaje para el procesamiento de la información de los alumnos.
- El modelo orientado al contexto, y que se dirige al estudio de la fenomenología de la conducta real de estudio y aprendizaje en las instituciones (Yussen, 1985).

Como se puede apreciar, el marco teórico se ha desarrollado de acuerdo con la concepción del modelo del procesamiento de la información.

\section{ESTUDIOS ANTECEDENTES}

En relación con las investigaciones realizadas con el Inventario de Estrategias de Aprendizaje y Estudio (IEAE), también conocido como LASSI por sus siglas en inglés, encontramos las siguientes contribuciones:

Strucchi (1991) realizó la adaptación psicométrica del Inventario de Estrategias de Aprendizaje y Estudio (IEAE) en la Argentina, para ello trabajó con una muestra de 850 estudiantes de la Universidad de Buenos Aires. Los resultados del análisis estadístico de las escalas indicaron que el instrumento presentó validez y confiabilidad.

Haught, Hill, Walls y Nardi (1998) desarrollaron un estudio con la finalidad de establecer el efecto de un programa de retroalimentación de los resultados en el Inventario de Estrategias de Aprendizaje y Estudio (IEAE) sobre el rendimiento académico, para ello trabajaron con 69 estudiantes en un semestre académico. La mitad de los participantes en un semestre llevaron un curso donde estudiaron sus perfiles 
individuales en el IEAE, recibiendo una retroalimentación individual y grupal, la otra mitad de los alumnos recibió una retroalimentación adicional consistente en sugerencias específicas para desarrollar sus estrategias.

Los resultados indicaron que los estudiantes que recibieron una mayor retroalimentación y consejería individual presentaron significativamente puntajes más altos al finalizar el curso en las escalas del IEAE de actitud, motivación, administración del tiempo, ansiedad, concentración, selección de ideas principales y estrategias para el examen.

Olaussen y Braten (1998) analizaron la estructura latente del IEAE. Para ello examinaron dos muestras independientes de estudiantes universitarios noruegos; la primera estuvo constituida por 173 estudiantes de ambos sexos de la Facultad de Educación de la Universidad de Oslo; en la segunda muestra participaron 176 estudiantes de ambos sexos, también de la Facultad de Educación.

Los análisis factoriales exploratorios realizados en las escalas del IEAE demostraron la existencia de la misma estructura latente de tres factores en las dos muestras. La estructura factorial fue similar a la reportada en los estudiantes universitarios americanos y los estudiantes de secundaria.

Los autores también realizaron análisis factoriales confirmatorios para examinar de qué manera los modelos de medida obtenidos en las investigaciones americanas se replicaban en los datos obtenidos en los alumnos noruegos. Sobre la base de dichos análisis, un modelo modificado fue generado, el cual fue probado en los datos de los estudiantes noruegos y americanos. Los tres constructos latentes fueron denominados como: actividades relacionadas con el esfuerzo, orientación hacia la meta y actividades cognoscitivas. Los resultados demostraron finalmente la capacidad de generalización transcultural de la estructura latente del LASSI.

Por otro lado, Olaussen y Braten (1999) realizaron el análisis de los resultados de algunos instrumentos actuales para determinar en los estudiantes el uso de estrategias de aprendizaje autorregulado. Los autores consideraron que las diferencias culturales que pueden contribuir a la diferenciación en el uso de dichas estrategias son la influencia del individualismo contra el colectivismo, el valor puesto en la educación, el valor de las creencias en la capacidad contra esfuerzo y de la ayuda social para el desarrollo académico.

También pusieron énfasis en los hallazgos obtenidos en un estudio previamente desarrollado por los mismos autores (Olaussen \& Braten, 1998). Ellos concluyeron que el uso de estrategias de aprendizaje autorregulado en los niveles posteriores a la secundaria demostraron una gran concordancia en 
Estados Unidos y Noruega y que el estudio americano sobre la autorregulación de los estudiantes debe, por lo tanto, ser altamente relevante para los educadores noruegos. Sin embargo, ponen énfasis en la necesidad de desarrollar un marco conceptual para entender los hallazgos paralelos y los contrastes de la autorregulación en los diversos grupos culturales.

Corral y Alcalá (2002) analizaron las dimensiones de las estrategias de aprendizaje y estudio, utilizando la versión argentina del IEAE; para ello trabajó con una muestra de 386 estudiantes del primer y del segundo año, en las cuatro carreras que se dictan en la Universidad Nacional del Nordeste de Argentina. Para ello realizó un análisis factorial que arrojó una estructura conformada por nueve factores o subescalas. La estructura fue examinada mediante el análisis del contenido de los ítems agrupados en cada uno de los nueve factores, a fin de caracterizarlos y determinar su sentido y alcance, lo que posibilitó algunas comparaciones con resultados obtenidos en otras poblaciones universitarias. Las autoras concluyen que la escala resultante en su estudio presentó diferencias sustanciales con respecto a la estructura factorial propuesta por Weinstein, Schulte y Palmer (1987).

En tanto que Flowers (2003) analizó la confiabilidad por estabilidad del inventario de estrategias y estudio (IEAE) en su versión para estudiantes de secundaria. El estudio tuvo por finalidad determinar el grado con el cual los puntajes de las escalas obtenidas en la primera edición del instrumento eran estables y permitían obtener medidas constantes del conocimiento y el uso de las habilidades para estudiar, para ello los resultados reportados por Weinstein (1987) se compararon con los resultados obtenidos en una muestra de 66 estudiantes con dificultades de ambos sexos y de diferentes etnias. Los resultados de las comparaciones realizadas permitieron observar que las puntuaciones del IEAE eran inconsistentes o inestables en el grupo de alumnos evaluados.

Finalmente, Sizoo, Malhotra y Bearson (2003) realizaron una comparación de las estrategias de aprendizaje evaluadas con el IEAE entre hombres y mujeres que estudiaban en la Facultad de Negocios, tanto entre aquellos con edades mayores de 25 años, considerados como alumnos adultos, y los menores de 25 años, considerados como alumnos tradicionales.

Los resultados indicaron que la muestra de mujeres alumnas adultas alcanzaron puntajes más elevados en motivación que los alumnos varones, tanto adultos como tradicionales, y que las mujeres alumnas tradicionales.

El análisis de la muestra de alumnos tradicionales (menores de 25 años) permitió observar que las mujeres presentaron mejores desempeños en las escalas de actitud, motivación, manejo del 
tiempo, concentración y ayudas para el estudio.

En lo que concierne al estudio de las estrategias de aprendizaje realizadas en nuestro contexto encontramos que las Escalas de Estrategias de Aprendizaje (ACRA) fue adaptada por Cano (1996) en el desarrollo de su tesis sobre las estrategias metacognitivas y cognitivas en el aprendizaje en alumnos del quinto de secundaria de NSE alto y medio alto en Lima metropolitana, obteniendo resultados psicométricos adecuados, pero a pesar de ello, y siguiendo la propuesta de Marín (1986), debido a la antigüedad del estudio se optó por realizar los análisis psicométricos respectivos de la validez y confiabilidad.

Por otro lado, García, Orellana y Canales (2002) evaluaron los factores que estaban asociados al rendimiento académico en los estudiantes de psicología de la Universidad Nacional Mayor de San Marcos, encontrando que existieron correlaciones negativas entre la orientación a metas intrínsecas y la ansiedad con el rendimiento académico y una asociación positiva entre la estrategia, la constancia y el rendimiento.

Aliaga y Giove (2003) analizaron las diferencias en el Cuestionario de Estrategias de Aprendizaje (MSLQ), y el IEAE entre los alumnos del quinto año de secundaria con autoevaluación alta y baja del rendimiento académico en matemática, observando que ocho de las 10 escalas del IEAE, 7 de las 12 escalas del MSLQ, la autoeficacia para el aprendizaje y la ansiedad ante los exámenes influyeron de forma significativa sobre el rendimiento.

Por último, Escurra, Delgado, Sotil, Pequeña, Quezada, Rivas, Solís y Santos (2004) estudiaron la influencia de las estrategias de aprendizaje y la reflexión activa sobre el rendimiento escolar de los alumnos de quinto año de secundaria de la ciudad de Lima. El estudio estuvo conformado por 1.200 adolescentes, se utilizó la escala ACRA para evaluar las estrategias de aprendizaje y se construyó una escala de reflexión activa.

Los hallazgos indicaron que las escalas ACRA y de reflexión activa presentaron validez y confiabilidad. Los puntajes de los alumnos en las áreas de la escala ACRA, la escala de reflexión activa y el rendimiento académico presentaron distribuciones que se aproximaron a la curva normal. Se observó que existieron diferencias significativas por tipo de gestión del colegio, pues los alumnos de colegios no estatales alcanzaron valores más elevados en las escalas de adquisición de la información, codificación de la información, recuperación de la información, apoyo al procesamiento y la reflexión activa.

También se apreció que existieron diferencias significativas por género, las mujeres presentaron mayores puntajes en las escalas de adquisición de la información, codificación de la infor- 
mación, recuperación de la información, apoyo al procesamiento y la reflexión activa.

Finalmente, concluyeron que las estrategias de aprendizaje y la reflexión activa influyen de manera positiva sobre el rendimiento escolar.

En la medida en que el instrumento que va a ser utilizado en la presente investigación implica su adaptación psicométrica, y siguiendo la propuesta de Marín (1986), se procederá a revisar el proceso de construcción y adaptación de pruebas psicológicas, pues existen diversos aspectos básicos que deben ser tomados en cuenta, como es el caso de la validez, la confiabilidad y las normas (Nunnally \& Bernstein, 1995; Cohen \& Swerdlik, 2001).

\section{Objetivos}

- Evaluar las estrategias de aprendizaje de los alumnos universitarios que estudian psicología.

- Adaptar el Inventario de Estrategias de Aprendizaje y Estudio (IEAE).

- Desarrollar las normas de aplicación y calificación del instrumento en una muestra representativa de alumnos universitarios que estudian psicología de Lima metropolitana.

- Establecer la confiabilidad por consistencia interna del Inventario de Estrategias de Aprendizaje y Estudio.
- Evaluar la validez de Constructo del Inventario de Estrategias de Aprendizaje y Estudio.

- Construir los baremos para el instrumento.

- Estudiar las diferencias en las estrategias de aprendizaje de los alumnos de psicología de Lima metropolitana, según las variables: género y tipo de universidad donde estudia.

\section{MÉTODO}

\section{Método de investigación}

La presente investigación se realizó en dos etapas. En la primera etapa se trabajó el Inventario de Estrategias de Aprendizaje y Estudio. De acuerdo con Kerlinger y Lee (2002), se trata de una investigación de tipo metodológico, en la medida en que se trata de evaluar la validez y confiabilidad de las pruebas estudiadas; en tanto que para Sánchez y Reyes (2002), la investigación también puede ser clasificada como aplicada, en la medida en que se orienta a la aplicación de manera práctica de los conocimientos teóricos de un determinado tema.

En la segunda etapa se estudiaron las características de las estrategias de aprendizaje de los alumnos universitarios que estudian psicología en Lima metropolitana, se trata de una investigación descriptiva (Alarcón, 1991; Salkind, 2000; Clark-Carter, 2002; 
Hernández, Fernández \& Baptista, 2003), debido a que permite la recolección de evidencias de hechos, concentrándose en el aquí y el ahora, como es el caso de las estrategias de aprendizaje de los estudiantes universitarios de psicología de Lima metropolitana.

\section{Diseño de investigación}

El presente estudio se realizó en dos etapas. En primer lugar se utilizó el diseño descriptivo simple (Hernández, Fernández \& Baptista, 2003), en el cual se estudiarán las características psicométricas del Inventario de Estrategias de Aprendizaje y Estudio.

En segundo lugar se utilizó el diseño descriptivo comparativo de grupos contrastantes (Alarcón, 1991; Sánchez \& Reyes, 2002; Hernández, Fernández \& Baptista, 2003) para comparar las estrategias de aprendizaje de acuerdo con las variables sociodemográficas: el tipo de gestión de la universidad donde estudia y el género de los alumnos.

\section{Población y muestra de estudio}

La población estuvo conformada por 2.500 alumnos universitarios que estudian psicología en las universidades estatales y particulares de Lima metropolitana.

a) Tamaño de la muestra

El tamaño de la muestra estimada ascendió a 566 casos, y fue calculada a través del programa SOTAM (Man- zano, 1997, 1998), en el cual se establecieron como parámetros un nivel de ocurrencia del $50 \%$, un margen de error del 5\% y un nivel de confianza bilateral del $99 \%(\mathrm{z}=2.58)$.

b) Muestreo

Dada la naturaleza del estudio, se desarrolló un muestreo estratificado polietápico (Lohr, 1999; Pérez, 2000), de acuerdo con el siguiente procedimiento:

En la primera etapa se construyó el marco muestral (Babbie, 2000), con el listado de los alumnos que estudian psicología en las universidades de Lima metropolitana.

En la segunda etapa, en cada universidad se estableció la fracción de la muestra correspondiente para mantener la representatividad de la muestra.

En la tercera etapa, en cada universidad seleccionada se procedió a sortear las aulas que van a ser evaluadas, según los ciclos que estudian. Y en dichas aulas se aplicaron los instrumentos respectivos.

c) Composición de la muestra

La composición de la muestra presentada en la tabla 1 indica que estuvo conformada por 566 alumnos universitarios estudiantes de psicología de Lima metropolitana, de los cuales el $47,7 \%$ provenían de universidades con gestión estatal y el $52,3 \%$ de universidades con gestión 
particular. Con respecto al género se observó que el 36,2\% fueron varones, en tanto que el $63,8 \%$ correspondió a mujeres.

Tabla 1

Composición de la muestra por género y tipo de gestión de la universidad

\begin{tabular}{lccc}
\hline \multirow{2}{*}{ Tipo de gestión } & \multicolumn{2}{c}{ Género } & Total \\
& Varón & Mujer & \\
\hline Nacional & 92 & 178 & 270 \\
& $16,3 \%$ & $31,4 \%$ & $47,7 \%$ \\
Particular & 113 & 183 & 296 \\
& $20,0 \%$ & $32,3 \%$ & $52,3 \%$ \\
Total & 205 & 361 & 566 \\
& $36,2 \%$ & $63,8 \%$ & $100,0 \%$ \\
\hline
\end{tabular}

Tabla 2

Composición general de la muestra de alumnos de psicología evaluados

\begin{tabular}{lcc}
\hline \multicolumn{1}{c}{ Variable } & $\mathrm{F}$ & $\%$ \\
\hline Edad & & \\
$\quad 18$ a 19 años & 156 & 27,6 \\
20 a 25 años & 327 & 57,8 \\
26 a 30 años & 93 & 14,6 \\
& & \\
Lugar de procedencia & & \\
$\quad$ Lima & 428 & 75,6 \\
$\quad$ Provincia & 138 & 24,4 \\
& & \\
Estado civil & & \\
$\quad$ Soltero(a) & 446 & 78,8 \\
$\quad$ Casado(a) & 120 & 21,2 \\
$\quad$ & & \\
Con quién vive & 11 & 1,9 \\
$\quad$ Solo & \\
$\quad$ Con su familia & 484 & 85,5 \\
$\quad$ Con otros parientes & 71 & 12,6 \\
\hline
\end{tabular}

$N=566$
En lo que concierne a la composición general de los alumnos, presentada en la tabla 2, se encuentra que los alumnos tuvieron entre 18 y 30 años, siendo la mayor proporción la correspondiente al grupo de 20 a 25 años $(57,8 \%)$.

Respecto al lugar de procedencia, la gran mayoría era de Lima $(75,6 \%)$, en tanto que el $24,4 \%$ era de provincias. En cuanto al estado civil, se apreció que el 78,8\% eran solteros(as), mientras que el $21,2 \%$ eran casados(as).

En relación con la pregunta con quién vive el alumno, se encontró que el $85,5 \%$ vivían con su familia, el $12,6 \%$ con otros parientes y solo el $1,9 \%$ vivían solos.

\section{INSTRUMENTO}

\section{Inventario de Estrategias de} Aprendizaje y Estudio (IEAE)

El Inventario de Estrategias de Aprendizaje y Estudio (IEAE), (LASSI, por sus siglas en inglés), fue inicialmente construido por Weinstein, Schulte y Palmer (1987) e incluye 77 ítems distribuidos en diez escalas (Weinstein \& Palmer, 2002), que se relacionan con cada uno de los tres componentes del aprendizaje estratégico: la habilidad, la voluntad y la autorregulación. Cada ítem es presentado como sobre la realización de una acción o actividad, de acuerdo con el formato tipo likert para ser calificado en una escala de cinco puntos respecto a la frecuencia de uso. 
La estructura conceptual del aprendizaje estratégico subyace en cada uno de estos componentes, existe algún traslape e interacción entre y dentro de los componentes y las escalas individuales. Sin embargo, los alumnos estratégicos necesitan saber sobre cada una de estas categorías y sobre cómo usar la información y las habilidades en cada una de ellas. Ellos también necesitan saber cómo escoger entre los varios elementos en cada categoría y ayudarlos a alcanzar metas de aprendizaje específicas y objetivos. Las escalas distribuidas de acuerdo con los componentes del aprendizaje estratégico son:

- El componente de habilidad del aprendizaje estratégico.- Las escalas que están relacionadas al componente de la habilidad del aprendizaje estratégico son el procesamiento de la información, la selección de ideas principales y la evaluación del rendimiento. Estas escalas permiten examinar las estrategias de aprendizaje de los estudiantes, las habilidades y el procesamiento del pensamiento, relacionado con la identificación, mientras van adquiriendo $\mathrm{y}$ construyendo el significado para la nueva información importante, las ideas y los procedimientos y la forma como ellos se preparan y demuestran sus nuevos conocimientos en pruebas $\mathrm{u}$ otros procedimientos de evaluación.

- La escala de procesamiento de la información evalúa cómo los es- tudiantes utilizan su imaginación, las elaboraciones verbales, las estrategias de la organización y las habilidades de razonamiento, así como las habilidades para aprender las estrategias con el fin de ayudar al aprendizaje de una nueva información y las habilidades y para construir relaciones entre ellas, de manera que el alumno puede saber que está intentando aprender y recordar.

- La escala de selección de ideas principales mide la habilidad de los estudiantes para identificar, durante un estudio extenso, la información importante de aquella que no lo es, así como los detalles de apoyo.

- La escala estrategia para el examen evalúa el uso, por parte de los estudiantes, de la forma como preparación de la prueba y las estrategias que utiliza para responder.

- El componente volitivo del aprendizaje estratégico.- Las escalas relacionadas al componente volitivo del aprendizaje estratégico son la ansiedad, la actitud y la motivación. Estas escalas miden el grado con que los estudiantes se preocupan por su desempeño académico, su receptividad para aprender la nueva información, sus actitudes e interés por la universidad, su diligencia, su autodisciplina y buena disposición para realizar el esfuerzo necesario pa- 
ra completar los requisitos académicos de forma completa.

- La escala de ansiedad mide el grado con que los estudiantes se preocupan por la universidad y su actuación académica.

- La escala de actitud evalúa las actitudes de los estudiantes y su interés por la universidad y el logro del éxito académico.

- La escala de motivación mide la diligencia de los estudiantes, la autodisciplina y la buena disposición para realizar el esfuerzo necesario para lograr o superar los requisitos académicos.

- El componente de autorregulación de aprendizaje estratégico.- Las escalas relacionadas con el componente de autorregulación del aprendizaje estratégico son: la concentración, la autoevaluación, las ayudas del estudio y el manejo del tiempo. Estas escalas miden cómo los estudiantes se controlan a sí mismos, o se autorregulan y controlan el proceso de aprendizaje a través del manejo eficaz del tiempo, mientras enfocan su atención y mantienen su concentración en el tiempo, verificando por sí mismos el cumplimiento de las demandas de aprendizaje de una clase, una asignación o una prueba, usando el estudio como apoyo, a través de sesiones de revisión, tutores o la identificación de rasgos especiales de un libro de texto.
- La escala de concentración evalúa la habilidad del estudiante para dirigir y mantener su atención en las tareas académicas.

- La escala de autoevaluación mide el uso, por los estudiantes, del repaso y la comprensión para supervisar las técnicas, con el fin de determinar su nivel de entendimiento de la información o el tema que debe ser aprendido.

- La escala de ayudas para el estudio mide el uso, por los estudiantes, de técnicas de apoyo, materiales o recursos para ayudarles a aprender y recordar la nueva información.

- La escala de administración del tiempo evalúa si los estudiantes utilizan principios para dirigir el tiempo asignado para las tareas académicas.

\section{Validez y confiabilidad de la prueba original}

La validez de constructo fue estudiada a través del análisis factorial exploratorio, el cual corroboró la existencia de las 10 escalas que constituyen el inventario (Weinstein \& Palmer, 2002).

La confiabilidad del inventario fue evaluada a través del coeficiente Alfa de Cronbach, computado para cada una de las escalas, obteniendo valores que fluctuaron entre 0.73 para la escala de ayudas para el estudio y 0.89 para la escala de selección de ideas principales. Estos 
resultados pueden ser considerados como adecuados (Weinstein \& Palmer, 2002). También se analizó la consistencia de los puntajes en el tiempo por medio del test-retest, alcanzando valores que fluctuaron entre 0.72 y 0.85 .

\section{Resultados}

A continuación se presentan los resultados alcanzados en los análisis estadísticos.

\section{Análisis psicométrico del Inventario de Estrategias de Aprendizaje y Estudio (IEAE)}

En primer lugar, se realizó el análisis de ítems y la confiabilidad de cada escala que compone el instrumento. En la tabla 3 se puede apreciar que los ítems obtuvieron correlaciones que son estadísticamente significativas $(\mathrm{p}<0.05)$, las cuales superan el criterio de $r>0.20$ propuesto por Kline (1995), por lo cual se puede afirmar que todos los ítems son relevantes para la escala.

La revisión de la confiabilidad por consistencia interna, calculado a través del coeficiente Alfa de Cronbach, indica que se obtiene un valor de 0.70 , por lo que se puede concluir que la escala de actitud permite obtener puntajes confiables, este hallazgo es corroborado por la estabilidad de los resultados presentados en el intervalo de confianza al 95\% del coeficiente Alfa de Cronbach, tal como lo sugieren Fan y Thompson (2001), Ledesma (2004) y Duhaheck y Iacobucci (2004).

El análisis de ítems de la escala de motivación (véase tabla 4) permite notar que se alcanzaron correlaciones que fluctúan entre $r=0.71$ y $r=0.76$, siendo en todos los casos estadísticamente significativas a $\mathrm{p}<.05$, por lo cual se puede indicar que todos los ítems son relevantes para la escala.

Tabla 3

Análisis de la confiabilidad de la escala de actitud

\begin{tabular}{cccc}
\hline İtem & Media & D. E. & Correlación ítem-test corregida \\
\hline P1ítem5 & 4.08 & 0.92 & $0.67^{*}$ \\
P1item14 & 3.71 & 0.99 & $0.68^{*}$ \\
P1ítem18 & 4.13 & 0.90 & $0.68^{*}$ \\
P1ítem29 & 3.45 & 0.96 & $0.68^{*}$ \\
P1item38 & 4.38 & 0.84 & $0.67^{*}$ \\
P1ítem45 & 3.51 & 1.07 & $0.68^{*}$ \\
P1ítem51 & 3.73 & 0.80 & $0.67^{*}$ \\
P1ítem69 & 3.92 & 0.94 & $0.66^{*}$ \\
\hline
\end{tabular}


Tabla 4

Análisis de la confiabilidad de la escala de actitud

\begin{tabular}{cccc}
\hline Ítem & Media & D. E. & Correlación ítem-test corregida \\
\hline P1ítem10 & 4.03 & 0.79 & $0.71^{*}$ \\
P1ítem13 & 3.66 & 0.90 & $0.76^{*}$ \\
P1ítem16 & 2.63 & 0.79 & $0.74^{*}$ \\
P1ítem28 & 3.87 & 0.94 & $0.70^{*}$ \\
P1ítem33 & 3.28 & 1.18 & $0.76^{*}$ \\
P1ítem41 & 4.06 & 0.79 & $0.71^{*}$ \\
P1ítem49 & 3.63 & 0.89 & $0.72^{*}$ \\
P1ítem56 & 3.71 & 0.85 & $0.75^{*}$ \\
\hline
\end{tabular}

Coeficiente Alfa de Cronbach $=0.76^{*}$

Intervalos de confianza al $95 \%$ del coeficiente Alfa de Cronbach $=0.73 ; 0.79$

* $p<.05$

$N=566$

Tabla 5

Análisis de la confiabilidad de la escala de administración del tiempo

\begin{tabular}{cccc}
\hline Ítem & Media & D. E. & Correlación ítem-test corregida \\
\hline P1ítem3 & 2.76 & 0.99 & $0.73^{*}$ \\
P1ítem22 & 2.44 & 0.85 & 0.76 * \\
P1ítem36 & 2.46 & 0.91 & $0.77^{*}$ \\
P1ítem42 & 2.78 & 0.98 & 0.74 * \\
P1ítem48 & 2.92 & 0.89 & 0.74 * \\
P1ítem58 & 3.35 & 0.99 & $0.79^{*}$ \\
P1ítem66 & 3.45 & 0.89 & 0.74 * \\
P1ítem74 & 3.62 & 0.98 & $0.77^{*}$ \\
\hline
\end{tabular}

Coeficiente Alfa de Cronbach $=0.78$ *

Intervalos de confianza al $95 \%$ del coeficiente Alfa de Cronbach $=0.76 ; 0.81$

${ }^{* p}<.05$

$\mathrm{N}=566$

El análisis de la confiabilidad por consistencia interna, calculado a través del coeficiente Alfa de Cronbach, asciende a 0.76 , por lo que se puede concluir que la escala de motivación es confiable.
El análisis de la escala de administración del tiempo, presentado en la tabla 5, permite apreciar que se obtuvieron correlaciones superiores a $\mathrm{r}=$ 0.72 , siendo en todos los casos estadísticamente significativas a $\mathrm{p}<.05$, lo 
que permite indicar que todos los ítems son relevantes para constituir la escala.

El análisis de la confiabilidad por consistencia interna, calculado a través del coeficiente Alfa de Cronbach, asciende a 0.78 , por lo que se puede concluir que la escala de administración del tiempo es confiable.

El análisis de ítems de la escala de ansiedad, presentado en la tabla 6 , indica que se alcanzaron correlaciones que fluctúan entre $r=0.70$ y r $=0.78$, siendo en todos los casos estadísticamente significativas a $\mathrm{p}<.05$, por lo cual se puede indicar que todos los ítems son relevantes para la escala.

El análisis de la confiabilidad por consistencia interna, calculado a través del coeficiente Alfa de Cronbach, asciende a 0.77 , por lo que se puede concluir que la escala de ansiedad es confiable.
En lo que concierne a los ítems de la escala de concentración, en la tabla 7 se encuentra que los ítems obtuvieron correlaciones que son estadísticamente significativas $(\mathrm{p}<0.05)$, las cuales superan el criterio de $r>0.20$ propuesto por Kline (1995), por lo cual se puede afirmar que todos los ítems son relevantes para conformar la escala.

La revisión de la confiabilidad por consistencia interna, calculado a través del coeficiente Alfa de Cronbach, indica que se obtiene un valor de 0.81 , por lo que se puede concluir que la escala de concentración permite obtener puntajes confiables.

El análisis de la escala de procesamiento de la información, presentado en la tabla 8 , permite notar que se obtuvieron correlaciones superiores a $\mathrm{r}=$ 0.62 , siendo en todos los casos estadísticamente significativas a $\mathrm{p}<.05$, lo

Tabla 6

Análisis de la confiabilidad de la escala de ansiedad

\begin{tabular}{|c|c|c|c|}
\hline Ítem & Media & D. E. & Correlación ítem-test corregida \\
\hline P1ítem1 & 1.80 & 0.90 & $0.78 *$ \\
\hline P1ítem9 & 2.27 & 1.05 & $0.77^{*}$ \\
\hline P1ítem25 & 3.87 & 0.96 & 0.73 * \\
\hline P1ítem31 & 2.83 & 1.15 & $0.71 *$ \\
\hline P1ítem35 & 3.68 & 0.80 & 0.77 * \\
\hline P1ítem54 & 3.56 & 0.94 & $0.70 *$ \\
\hline P1ítem57 & 3.17 & 1.20 & $0.70 *$ \\
\hline P1ítem63 & 3.67 & 1.03 & 0.71 * \\
\hline \multicolumn{4}{|c|}{$\begin{array}{l}\text { Coeficiente Alfa de Cronbach }=0.77{ }^{*} \\
\text { fianza al } 95 \% \text { del coeficiente Alfa de Cronbach }=0.74 ; 0.80\end{array}$} \\
\hline
\end{tabular}


que permite indicar que todos los ítems son relevantes para constituir la escala.

El análisis de la confiabilidad por consistencia interna, calculado a través del coeficiente Alfa de Cronbach, asciende a 0.72 , por lo que se puede concluir que la escala de procesamiento de la información es confiable.
El análisis de ítems de la escala de selección de ideas principales, incluido en la tabla 9, se encuentran correlaciones superiores a $r=0.70$, siendo en todos los casos estadísticamente significativas a $\mathrm{p}<.05$, por lo cual se puede indicar que todos los ítems son relevantes para la escala.

Tabla 7

Análisis de la confiabilidad de la escala de concentración

\begin{tabular}{cccc}
\hline Ítem & Media & D. E. & Correlación ítem-test corregida \\
\hline P1ítem6 & 3.41 & 0.71 & $0.79^{*}$ \\
P1ítem11 & 3.12 & 0.94 & $0.80^{*}$ \\
P1ítem39 & 2.97 & 0.77 & $0.80^{*}$ \\
P1ítem43 & 3.29 & 0.84 & $0.78^{*}$ \\
P1ítem46 & 2.76 & 1.06 & $0.75^{*}$ \\
P1ítem55 & 3.22 & 0.86 & $0.79^{*}$ \\
P1ítem61 & 3.38 & 0.93 & $0.79^{*}$ \\
P1ítem68 & 3.03 & 0.98 & $0.75^{*}$ \\
\hline
\end{tabular}

Coeficiente Alfa de Cronbach $=0.81^{*}$ Intervalos de confianza al $95 \%$ del coeficiente Alfa de Cronbach $=0.78 ; 0.83$

$* p<.05$

$\mathrm{N}=566$

Tabla 8

Análisis de la confiabilidad de la escala de procesamiento de información

\begin{tabular}{|c|c|c|c|}
\hline Ítem & Media & D. E. & Correlación ítem-test corregida \\
\hline P1ítem12 & 3.39 & 0.96 & $0.72 *$ \\
\hline P1ítem15 & 3.67 & 0.89 & 0.71 * \\
\hline P1ítem23 & 3.84 & 0.87 & 0.71 * \\
\hline P1ítem32 & 4.19 & 0.75 & 0.68 * \\
\hline P1ítem40 & 3.94 & 0.72 & $0.66 *$ \\
\hline P1ítem47 & 3.87 & 0.93 & 0.63 * \\
\hline P1ítem67 & 3.55 & 0.98 & 0.68 * \\
\hline P1ítem76 & 3.70 & 0.87 & $0.65 *$ \\
\hline Interva & $\begin{array}{l}\text { Coeficie } \\
\text { ianza al }\end{array}$ & Cronba & Cronbach $=0.68 ; 0.75$ \\
\hline
\end{tabular}


Tabla 9

Análisis de la confiabilidad de la escala de selección de ideas principales

\begin{tabular}{cccc}
\hline Ítem & Media & D. E. & Correlación ítem-test corregida \\
\hline P1ítem2 & 3.92 & 0.69 & $0.77^{*}$ \\
P1ítem8 & 4.03 & 0.81 & $0.83^{*}$ \\
P1ítem60 & 3.75 & 0.86 & $0.74^{*}$ \\
P1ítem72 & 3.73 & 0.82 & $0.73^{*}$ \\
P1ítem77 & 3.70 & 0.89 & $0.71^{*}$ \\
\hline
\end{tabular}

Coeficiente Alfa de Cronbach $=0.80$ * Intervalos de confianza al $95 \%$ del coeficiente Alfa de Cronbach $=0.77 ; 0.83$

$* p<.05$

$\mathrm{N}=566$

El análisis de la confiabilidad por consistencia interna, calculado a través del coeficiente Alfa de Cronbach, asciende a 0.80 , por lo que se puede concluir que la escala de selección de ideas principales es confiable.

El análisis de la escala de ayudas para el estudio, incluido en la tabla 10, permite observar que se alcanzaron correlaciones superiores a $r=0.60$, siendo en todos los casos estadísticamente significativas a $\mathrm{p}<.05$, lo que permite indicar que todos los ítems son relevantes para constituir la escala.

El análisis de la confiabilidad por consistencia interna, calculado a través del coeficiente Alfa de Cronbach, asciende a 0.73 , por lo que se puede concluir que la escala de ayudas para el estudio es confiable.

En lo que respecta al análisis de la escala de autoevaluación, presentado en la tabla 11, se puede apreciar que alcanzaron correlaciones superiores a

Tabla 10

Análisis de la confiabilidad de la escala

\begin{tabular}{cccc}
\hline ítem & Media & D. E. & Correlación ítem-test corregida \\
\hline p1ítem7 & 4.21 & 0.86 & 0.69 * \\
P1ítem19 & 3.90 & 0.90 & $0.70^{*}$ \\
P1ítem24 & 3.21 & 1.21 & $0.72 *$ \\
P1ítem44 & 4.23 & 0.82 & $0.70^{*}$ \\
P1ítem50 & 3.68 & 1.11 & $0.64^{*}$ \\
P1ítem53 & 3.40 & 1.18 & $0.611^{*}$ \\
P1ítem62 & 3.62 & 0.97 & 0.72 * \\
P1ítem73 & 2.79 & 1.02 & $0.73^{*}$ \\
\hline
\end{tabular}

Coeficiente Alfa de Cronbach $=0.73$

Intervalos de confianza al 95\% del coeficiente Alfa de Cronbach $=0.69 ; 0.76$ 
Tabla 11

Análisis de la confiabilidad de la escala de autoevaluación

\begin{tabular}{cccc}
\hline ítem & Media & D. E. & Correlación ítem-test corregida \\
\hline P1ítem4 & 2.47 & 0.88 & $0.65^{*}$ \\
P1ítem17 & 3.58 & 0.98 & $0.622^{*}$ \\
P1ítem21 & 3.70 & 0.99 & $0.63^{*}$ \\
P1ítem26 & 2.25 & 0.82 & $0.63^{*}$ \\
P1ítem30 & 3.68 & 0.94 & $0.64^{*}$ \\
P1ítem37 & 3.62 & 0.87 & $0.65^{*}$ \\
P1ítem65 & 3.64 & 0.95 & $0.63^{*}$ \\
P1ítem70 & 3.05 & 0.93 & $0.62^{*}$ \\
\hline
\end{tabular}

Coeficiente Alfa de Cronbach $=0.70$ *

Intervalos de confianza al $95 \%$ del coeficiente Alfa de Cronbach $=0.66 ; 0.75$

${ }^{*} \mathrm{p}<.05$

$\mathrm{N}=566$

$\mathrm{r}=0.61$, siendo en todos los casos estadísticamente significativas a $\mathrm{p}<.05$, lo que permite indicar que todos los ítems son relevantes para constituir la escala.

El análisis de la confiabilidad por consistencia interna, calculado a través del coeficiente Alfa de Cronbach, as- ciende a 0.70 , por lo que se puede concluir que la escala de autoevaluación es confiable.

El análisis de ítems de la escala de estrategias para el examen (véase tabla 12), permite notar que se alcanzaron correlaciones que fluctúan entre $r=0.71 \mathrm{y}$ $r=0.76$, siendo en todos los casos es-

Tabla 12

Análisis de la confiabilidad de la escala de estrategias para el examen

\begin{tabular}{cccc}
\hline Ítem & Media & D. E. & Correlación ítem-test corregida \\
\hline P1ítem20 & 3.27 & 0.94 & $0.78^{*}$ \\
P1ítem27 & 3.97 & 0.92 & $0.75^{*}$ \\
P1ítem34 & 3.49 & 0.98 & $0.75^{*}$ \\
P1ítem52 & 4.00 & 0.68 & $0.77^{*}$ \\
P1ítem59 & 4.17 & 0.92 & $0.77^{*}$ \\
P1ítem64 & 3.57 & 1.01 & $0.78^{*}$ \\
P1ítem71 & 3.34 & 0.87 & $0.75^{*}$ \\
P1ítem75 & 3.77 & 0.89 & $0.75^{*}$ \\
\hline
\end{tabular}

Coeficiente Alfa de Cronbach $=0.79$ *

Intervalos de confianza al $95 \%$ del coeficiente Alfa de Cronbach $=0.76 ; 0.82$ 
tadísticamente significativas a $\mathrm{p}<.05$, por lo cual se puede indicar que todos los ítems son relevantes para la escala.

El análisis de la confiabilidad por consistencia interna, calculado a través del coeficiente Alfa de Cronbach, asciende a 0.79 , por lo que se puede concluir que la escala de estrategias para el examen es confiable.

El análisis de la confiabilidad generalizado, que incluye a las escalas del IEAE como ítems, presentado en la tabla 13, permite observar que se alcanzan correlaciones que son superiores a $r=0.84$ y que son en todos los casos estadísticamente significativos, lo que nos indica que todas las escalas son consistentes entre sí.
El análisis de la confiabilidad de las escalas indica que se obtuvo un coeficiente Alfa de Cronbach de 0.87, por lo que se concluye que el IEAE es confiable.

El estudio de la validez de constructo fue realizado a través del análisis factorial confirmatorio, aplicándose los programas Lisrel 8.54 (Jöreskog \& Sörbom, 2004) y Amos 5.0 (Arbuckle, 2004). Los resultados alcanzados en el modelo teórico propuesto de un factor, de acuerdo con las recomendaciones propuestas por Byrne $(1998,2001)$ se contrastó con un modelo alternativo.

Los hallazgos del análisis de las escalas del IEAE (véanse tabla 14 y gráfico 1), permiten apreciar que el mode-

Tabla 13

Análisis generalizado de la confiabilidad del IEAE

\begin{tabular}{lccc}
\hline \multicolumn{1}{c}{ Ítem } & Media & D. E. & $\begin{array}{c}\text { Correlación ítem-test } \\
\text { corregida }\end{array}$ \\
\hline Actitud & 31.13 & 4.30 & $0.85^{*}$ \\
Motivación & 29.17 & 4.47 & $0.85^{*}$ \\
Administración del tiempo & 24.19 & 4.97 & $0.85^{*}$ \\
Ansiedad & 25.15 & 5.01 & $0.88^{*}$ \\
Concentración & 25.64 & 4.89 & $0.85^{*}$ \\
Procesamiento de información & 30.25 & 4.09 & $0.86^{*}$ \\
Seleccción de ideas principales & 19.28 & 3.11 & $0.85^{*}$ \\
Ayudas para el estudio & 29.15 & 4.80 & $0.86^{*}$ \\
Autoevaluación & 26.34 & 4.27 & $0.86^{*}$ \\
Estrategia para el examen & 29.68 & 4.67 & $0.85^{*}$ \\
\hline
\end{tabular}

Coeficiente Alfa de Cronbach $=0.87$ *

Intervalos de confianza al 95\% del coeficiente Alfa de Cronbach $=0.86 ; 0.89$

$* p<.05$

$N=566$ 
lo de tres factores que constituyen los componentes del aprendizaje estratégico presentan, en el test de bondad de ajuste Chi-cuadrado mínimo (Cmin) un valor de 19.38, el cual con 13 grados de libertad alcanza una probabilidad de 0.112 , lo que nos indica que el modelo es adecuado.

Tabla 14

Análisis de la validez de constructo del IEAE a través del análisis factorial confirmatorio

\begin{tabular}{lcc}
\hline Datos & $\begin{array}{c}\text { Modelo de } \\
\text { 3 factores }\end{array}$ & $\begin{array}{c}\text { Modelo } \\
\text { independiente }\end{array}$ \\
\hline $\begin{array}{l}\text { Parámetros } \\
\text { Chi-cuadrado }\end{array}$ & 42 & 10 \\
$\quad$ mínimo & 19.38 & 1195.82 \\
G. L. & 13 & 45 \\
P & 0.112 & 0.000 \\
Chi-cuadrado & & \\
$\quad$ mínimo/G.L. & 1.49 & 26.57 \\
RMR & 0.04 & 8.01 \\
GFI & 0.98 & 0.37 \\
AGFI & 0.93 & 0.23 \\
RMSEA & 0.02 & 2.58 \\
\hline
\end{tabular}

$\mathrm{N}=566$

La revisión de los hallazgos de los análisis del índice residual de la raíz cuadrada media de los residuales que evalúa la aproximación de la matriz de covarianzas teórica con la matriz observada $(R M R=0.04)$ y la raíz cuadrada media del error de aproximación $($ RMSEA $=0.02)$ presentan valores pequeños, además los análisis complementarios de la bondad de ajuste a través del índice de ajuste $(\mathrm{GFI}=0.98)$ y el índice de ajuste ponderado (AGFI = 0.93 ) alcanzaron valores óptimos, estos hallazgos permitieron aceptar el modelo de tres factores. Por lo que se concluye que el IEAE presenta validez de constructo.

\section{Gráfico 1}

Modelo de ecuaciones estructurales de la validez de constructo del IEAE

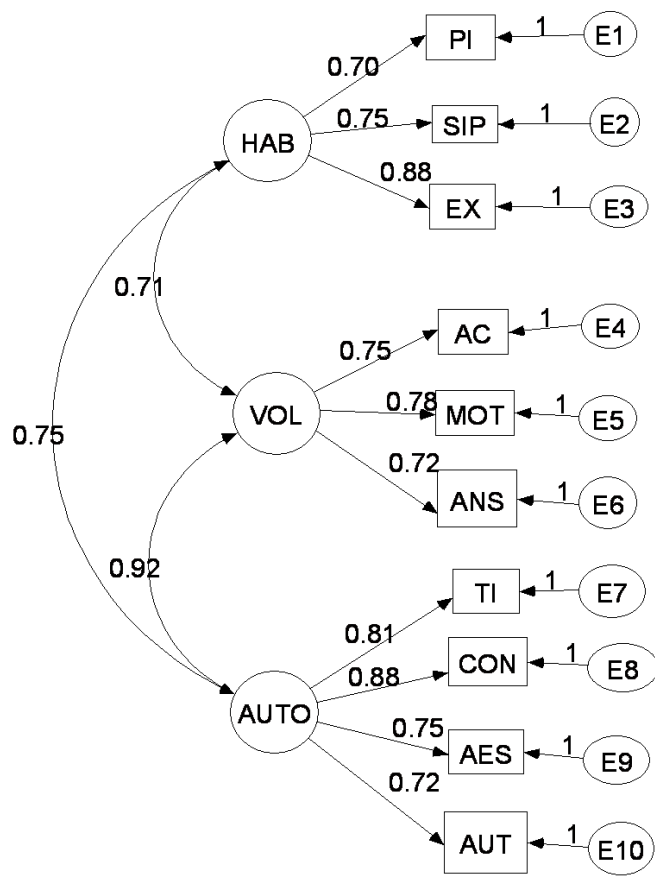

Análisis exploratorio de las variables estudiadas

El análisis de la bondad ajuste a la curva normal de las escalas del IEAE, realizado a través de la prueba de Kolmogorov-Smirnov (véase tabla 15), permite apreciar que en todas las escalas se encuentran resultados que no son 
significativos, por lo que se puede concluir que los puntajes de las escalas se distribuyen de acuerdo con una curva normal.

Tabla 15

Análisis de la aproximación a la curva normal de las escalas del IEAE a través de la prueba de Kolmogorov-Smirnov

\begin{tabular}{lccc}
\hline Item & Media & D. E. & K-S Z \\
\hline Actitud & 3.89 & 0.54 & 1.36 \\
Motivación & 3.65 & 0.56 & 1.58 \\
$\begin{array}{l}\text { Administración } \\
\quad \text { del tiempo }\end{array}$ & 3.02 & 0.62 & 1.25 \\
$\begin{array}{l}\text { Ansiedad } \\
\text { Concentración }\end{array}$ & 3.14 & 0.63 & 1.30 \\
$\begin{array}{l}\text { Procesamiento } \\
\text { de información }\end{array}$ & 3.21 & 0.61 & 1.66 \\
$\begin{array}{l}\text { Selección de ideas } \\
\text { principales }\end{array}$ & 3.78 & 0.511 .77 & \\
$\begin{array}{l}\text { Ayudas para el } \\
\quad \text { estudio }\end{array}$ & 3.86 & 0.621 .05 & \\
$\begin{array}{l}\text { Autoevaluación } \\
\text { Estrategia para el } \\
\text { examen }\end{array}$ & 3.64 & 0.60 & 1.92 \\
\hline & 3.71 & 0.53 & 1.13 \\
\hline
\end{tabular}

$N=566$

En lo que concierne al análisis de la bondad ajuste a la curva normal de los componentes del aprendizaje estratégico del IEAE, realizado a través de la prueba de Kolmogorov-Smirnov (véase tabla 16), permite apreciar que en todos los componentes se encuentran resultados que no son significativos, por lo que se puede concluir que los puntajes se distribuyen de acuerdo con una curva normal.

\section{Tabla 16}

Análisis de la aproximación a la curva normal de los componentes del aprendizaje estratégico del IEAE a través de la prueba de Kolmogorov-Smirnov

\begin{tabular}{lccc}
\hline Factor & Media & D. E. & K-S Z \\
\hline Habilidad & 3.78 & 0.48 & 1.55 \\
Volitivo & 3.56 & 0.42 & 1.15 \\
Autorregulación & 3.29 & 0.45 & 1.10 \\
\hline
\end{tabular}

$p<.05$

$\mathrm{N}=566$

Análisis comparativo según variables independientes asignadas

Con relación a la comparación de las escalas del IEAE según el tipo de gestión de la universidad realizado a través de la prueba $\mathrm{Z}$ de diferencia de medias, el cual es presentado en la tabla 17 , permite observar que existen diferencias estadísticas significativas a favor de las universidades particulares, en actitud $(Z=-$ $2.28, \mathrm{p}<.05)$, motivación $(\mathrm{Z}=-2.91$, $\mathrm{p}<.01)$ y autoevaluación $(\mathrm{Z}=-1.97$, $\mathrm{p}<.05)$.

Con la finalidad de obtener una evaluación más completa de los análisis estadísticos realizados, se calculó el tamaño del efecto, es decir cuán grandes son las diferencias que existen entre los grupos comparados y la potencia estadística de las pruebas utilizadas, es decir la probabilidad de evitar un error tipo II, que corresponde a la probabilidad de aceptar una hipótesis nula cuando realmente es cierta (Cohen, 1988, 1992; Aron \& Aron, 2001; ClarkCarter, 2002). 
Tabla 17

Prueba $Z$ de diferencia de medias de las escalas del IEAE por tipo de gestión de la universidad

\begin{tabular}{|c|c|c|c|c|c|c|c|}
\hline \multirow[t]{2}{*}{ Escalas } & \multicolumn{2}{|c|}{$\begin{array}{l}\text { Nacional } \\
\mathrm{N}=270\end{array}$} & \multicolumn{2}{|c|}{$\begin{array}{l}\text { Particular } \\
N=296\end{array}$} & \multirow[t]{2}{*}{ Z } & \multirow[t]{2}{*}{$d$} & \multirow[t]{2}{*}{ Poder } \\
\hline & $\mathrm{M}$ & D. E. & $\mathrm{M}$ & D. E. & & & \\
\hline $\begin{array}{l}\text { Actitud } \\
\text { Motivación }\end{array}$ & $\begin{array}{l}3.84 \\
3.58\end{array}$ & $\begin{array}{l}0.53 \\
0.55\end{array}$ & $\begin{array}{l}3.94 \\
3.71\end{array}$ & $\begin{array}{l}0.54 \\
0.56\end{array}$ & $\begin{array}{l}-2.28 * \\
-2.91 \text { ** }\end{array}$ & $\begin{array}{l}0.19 \\
0.23\end{array}$ & $\begin{array}{l}0.62 \\
0.78\end{array}$ \\
\hline $\begin{array}{l}\text { Administración del } \\
\text { tiempo }\end{array}$ & 3.00 & 0.59 & 3.05 & 0.65 & -0.96 & 0.08 & 0.16 \\
\hline $\begin{array}{l}\text { Ansiedad } \\
\text { Concentración }\end{array}$ & $\begin{array}{l}3.17 \\
3.17\end{array}$ & $\begin{array}{l}0.60 \\
0.58\end{array}$ & $\begin{array}{l}3.12 \\
3.23\end{array}$ & $\begin{array}{l}0.65 \\
0.64\end{array}$ & $\begin{array}{r}0.95 \\
-1.15\end{array}$ & $\begin{array}{l}0.08 \\
0.10\end{array}$ & $\begin{array}{l}0.16 \\
0.22\end{array}$ \\
\hline $\begin{array}{l}\text { Procesamiento de } \\
\text { información }\end{array}$ & 3.76 & 0.51 & 3.80 & 0.51 & -0.92 & 0.08 & 0.16 \\
\hline $\begin{array}{l}\text { Selección de ideas } \\
\text { principales }\end{array}$ & 3.85 & 0.63 & 3.86 & 0.62 & -0.35 & 0.02 & 0.06 \\
\hline Ayudas para el & 3.62 & 0.61 & 3.67 & 0.59 & -0.98 & 0.08 & 0.16 \\
\hline Autoevaluación & 3.25 & 0.53 & 3.34 & 0.54 & $-1.97^{*}$ & 0.17 & 0.52 \\
\hline $\begin{array}{l}\text { Estrategia para el } \\
\text { examen }\end{array}$ & 3.69 & 0.59 & 3.73 & 0.58 & -0.98 & 0.07 & 0.13 \\
\hline
\end{tabular}

$* p<.05 * * p<.01$

Los hallazgos presentados en la tabla 17 permiten apreciar que el tamaño del efecto de las diferencias en los tipos de gestión de la universidad alcanzaron valores bajos en la actitud $(\mathrm{d}=0.19)$, la motivación $(\mathrm{d}=0.23)$ y la autoevaluación $(\mathrm{d}=0.17)$, lo cual implica que las diferencias son significativas y adecuados en el estudio. En tanto que en el análisis del poder estadístico se aprecia que la motivación alcanza un nivel de 0.78 , en tanto que en actitud a 0.62 y la autoevaluación de 0.52 .

Con relación a la comparación de las diferencias de los componentes del aprendizaje estratégico por tipo de gestión de la universidad, presentado en la tabla 18, se encuentra que no existen diferencias estadísticas significativas en caso alguno.

Tabla 18

Prueba $Z$ de diferencia de medias de los componentes del aprendizaje estratégico del IEAE por tipo de gestión de la universidad

\begin{tabular}{|c|c|c|c|c|c|c|c|}
\hline \multirow[t]{2}{*}{ Componentes } & \multicolumn{2}{|c|}{$\begin{array}{c}\text { Nacional } \\
\mathrm{N}=270\end{array}$} & \multicolumn{2}{|c|}{$\begin{array}{c}\text { Particular } \\
N=296\end{array}$} & \multirow[t]{2}{*}{ Z } & \multirow[t]{2}{*}{$d$} & \multirow[t]{2}{*}{ Poder } \\
\hline & $\mathrm{M}$ & D. E. & $\mathrm{M}$ & D. E. & & & \\
\hline Habilidad & 3.76 & 0.49 & 3.80 & 0.47 & -0.96 & 0.08 & 0.16 \\
\hline Volitivo & 3.53 & 0.42 & 3.59 & 0.41 & -1.80 & 0.14 & 0.38 \\
\hline Autorregulación & 3.26 & 0.44 & 3.32 & 0.46 & -1.64 & 0.13 & 0.34 \\
\hline
\end{tabular}


Tabla 19

Prueba $Z$ de diferencia de medias de las escalas del IEAE por género

\begin{tabular}{|c|c|c|c|c|c|c|c|}
\hline \multirow{2}{*}{ Escalas } & \multicolumn{2}{|c|}{$\begin{array}{l}\text { Hombre } \\
\mathrm{N}=205\end{array}$} & \multicolumn{2}{|c|}{$\begin{array}{c}\text { Mujer } \\
N=361\end{array}$} & \multirow[t]{2}{*}{ Z } & \multirow[t]{2}{*}{$d$} & \multirow[t]{2}{*}{ Poder } \\
\hline & $M$ & D. E. & $M$ & D. E. & & & \\
\hline Actitud & 3.83 & 0.55 & 3.93 & 0.53 & $-2.02^{*}$ & 0.19 & 0.58 \\
\hline Motivación & 3.60 & 0.58 & 3.67 & 0.55 & -1.56 & 0.12 & 0.28 \\
\hline $\begin{array}{l}\text { Administración del } \\
\text { tiempo }\end{array}$ & 2.94 & 0.66 & 3.07 & 0.60 & $-2.51^{*}$ & 0.21 & 0.67 \\
\hline Ansiedad & 3.06 & 0.63 & 3.29 & 0.59 & $-4.11 * * *$ & 0.37 & 0.99 \\
\hline Concentración & 3.14 & 0.68 & 3.24 & 0.57 & -1.89 & 0.16 & 0.45 \\
\hline $\begin{array}{l}\text { Procesamiento de } \\
\text { información }\end{array}$ & 3.85 & 0.50 & 3.74 & 0.51 & $2.44^{* *}$ & 0.22 & 0.71 \\
\hline $\begin{array}{l}\text { Selección de ideas } \\
\text { principales }\end{array}$ & 3.86 & 0.71 & 3.85 & 0.57 & 0.10 & 0.02 & 0.06 \\
\hline Ayudas para el estudio & 3.51 & 0.65 & 3.72 & 0.56 & $-4.20 * * *$ & 0.35 & 0.97 \\
\hline Autoevaluación & 3.28 & 0.60 & 3.30 & 0.49 & -0.59 & 0.04 & 0.07 \\
\hline $\begin{array}{l}\text { Estrategia para el } \\
\text { examen }\end{array}$ & 3.74 & 0.62 & 3.69 & 0.56 & 0.90 & 0.09 & 0.18 \\
\hline
\end{tabular}

$* p<.05 * * p<.01 * * * 0<.001$

En lo que concierne a la comparación de las escalas del IEAE según el género, efectuada a través de la prueba $Z$ de diferencia de medias, la cual es presentada en la tabla 19, permite observar que existen diferencias estadísticas significativas a favor de las mujeres en actitud $(Z=-2.02, p<.05)$, tiempo $(Z=-2.51, \mathrm{p}<.05)$, ansiedad $(Z=-4.11, \mathrm{p}<.001)$ y ayudas para el estudio $(Z=-4.20, p<.001)$. En tanto que los hombres superan a las mujeres en la escala de procesamiento de la información $(Z=2.44, p<.01)$.

Los hallazgos presentados en la tabla 19, permiten apreciar que el tamaño del efecto, de las diferencias según género alcanzaron un valor medio en Ansiedad $(\mathrm{d}=0.37)$ y alcanzaron valores bajos en Actitud $(d=0.19)$, la Administración del Tiempo $(\mathrm{d}=0.21)$ y el Procesamiento de la Información $(\mathrm{d}=0.21)$, lo cual implica que las diferencias son significativas. En tanto que en el análisis del poder estadístico se aprecia que la Ansiedad alcanza un nivel de 0.99, en tanto que en el Procesamiento de la Información asciende a 0.71 , la Administración del Tiempo es de 0.67 y la Actitud a 0.58 .

Con relación a la comparación de las diferencias de los componentes del aprendizaje estratégico por género, presentado en la tabla 20 , se encuentra que existen diferencias estadísticas significativas a favor de las mujeres en el componente del aprendizaje estratégico de autorregulación $(Z=-3.09$, $\mathrm{p}<.001)$. 
Tabla 20

Prueba $Z$ de diferencia de medias de los componentes del aprendizaje estratégico del IEAE por género

\begin{tabular}{|c|c|c|c|c|c|c|c|}
\hline \multirow[t]{2}{*}{ Factores } & \multicolumn{2}{|c|}{$\begin{array}{l}\text { Hombre } \\
\mathrm{N}=205\end{array}$} & \multicolumn{2}{|c|}{$\begin{array}{c}\text { Mujer } \\
\mathrm{N}=361\end{array}$} & \multirow[t]{2}{*}{ Z } & \multirow[t]{2}{*}{$d$} & \multirow[t]{2}{*}{ Poder } \\
\hline & $\mathrm{M}$ & D. E. & $\mathrm{M}$ & D. E. & & & \\
\hline Habilidad & 3.82 & 0.52 & 3.76 & 0.45 & 1.47 & 0.13 & 0.32 \\
\hline Volitivo & 3.57 & 0.41 & 3.55 & 0.42 & 0.47 & 0.05 & 0.09 \\
\hline Autorregulación & 3.21 & 0.49 & 3.34 & 0.42 & -3.09 * & 0.29 & 0.91 \\
\hline
\end{tabular}

$* p<.001$

Los hallazgos presentados en la tabla 20 permiten apreciar que el tamaño del efecto de las diferencias según género alcanzaron un valor medio en autorregulación $(\mathrm{d}=0.29)$. En tanto que en el análisis del poder estadístico se aprecia que la autorregulación alcanza un nivel de 0.91 .
Análisis del grado de desarrollo de las escalas y de los componentes del aprendizaje estratégico del IEAE

En el análisis del grado de desarrollo de las escalas en la muestra evaluada, realizado a través de la prueba $\mathrm{Z}$ de una muestra y tomando el valor de 3 como valor promedio esperado. En la tabla 21

Tabla 21

Análisis del grado de desarrollo de las escalas del IEAE

\begin{tabular}{lccc}
\hline \multicolumn{1}{c}{ Escalas } & Media & D. E. & Z \\
\hline Actitud & 3.89 & 0.54 & $39.45^{*}$ \\
Motivación & 3.65 & 0.56 & $27.50^{*}$ \\
Administración del tiempo & 3.02 & 0.62 & 0.91 \\
Ansiedad & 3.14 & 0.63 & $5.46^{*}$ \\
Concentración & 3.21 & 0.61 & $7.97^{*}$ \\
Procesamiento de información & 3.78 & 0.51 & $36.34^{*}$ \\
Selección de ideas principales & 3.86 & 0.62 & $32.74^{*}$ \\
Ayudas para el estudio & 3.64 & 0.60 & $25.53^{*}$ \\
Autoevaluación & 3.29 & 0.53 & $13.04^{*}$ \\
Estrategia para el examen & 3.71 & 0.58 & $28.93^{*}$ \\
\hline
\end{tabular}

$* p<.001$

Valor de comparación $=3$

$N=566$ 
se aprecia que salvo en la escala de tiempo, en todas las demás escalas del IEAE se encuentran resultados que son estadísticamente significativos, es decir que en general las escalas se encuentran desarrollados por encima del valor promedio esperado, por lo que se puede concluir que los puntajes de las escalas se encuentran adecuadamente desarrolladas en los alumnos estudiados.

Tabla 22

Análisis del grado de desarrollo de los componentes del aprendizaje estratégico del IEAE

\begin{tabular}{lccc}
\hline Componente & Media & D. E. & Z \\
\hline Habilidad & 3.78 & 0.48 & 38.88 * \\
Volitivo & 3.56 & 0.42 & 31.92 * \\
Autorregulación & 3.29 & 0.45 & 15.48 *
\end{tabular}

${ }^{*} \mathrm{p}<.001$

Valor de comparación $=3$

$\mathrm{N}=566$

En el análisis del grado de desarrollo de los componentes del aprendizaje estratégico en la muestra evaluada, realizado a través de la prueba $\mathrm{Z}$ de una muestra y tomando el valor de 3 como valor promedio esperado, en la tabla 22 , se aprecia que en todos los casos se encuentran diferencias estadísticas significativas, es decir que en general los componentes se encuentran desarrollados por encima del valor promedio esperado, por lo que se puede concluir que los puntajes de los componentes del aprendizaje estratégico se encuentran adecuadamente desarrollados en los alumnos estudiados.

\section{Análisis complementarios}

Con la finalidad de generar una tipología entre los factores asociados a las habilidades sociales, se realizó un análisis de Cluster a través del método de $\mathrm{K}$ medias (Hair, Anderson, Tatham \& Black, 1999; Johnson, 2000; D'Ancona, 2002), para el desarrollo del análisis se propuso la existencia de una clasificación en tres grupos (véase tabla 23).

Los resultados del análisis de Cluster corroboraron la existencia de tres grupos diferenciados, lo cual se puede apreciar en la tabla 24, donde se incluyen las comparaciones de los valores de los tres grupos, notándose que todas las escalas del IEAE incluidas presentan diferencias estadísticas significativas.

En la tabla 24 se presentan los promedios obtenidos por los grupos identificados, y en el gráfico 2 se presentan los perfiles característicos de cada uno de los grupos. La revisión de estos resultados nos permite apreciar lo siguiente:

El Cluster 1 está conformado por 180 casos e incluye a los alumnos que presentan los puntajes más altos en las escalas del IEAE, por lo que se trataría de un grupo con un perfil que podríamos denominarlo como de "Estrategias Elevadas". 
Tabla 23

Análisis de Cluster, evaluación de la significación de las escalas del IEAE incluidas

\begin{tabular}{lccc}
\hline \multirow{2}{*}{ Escalas } & \multicolumn{2}{c}{ Cluster } & Error \\
\cline { 2 - 3 } & Media de cuadrados & $\begin{array}{c}\text { Media de } \\
\text { cuadrados }\end{array}$ & $\mathrm{F}$ \\
\hline Actitud & 37.85 & 0.16 & $243.36^{*}$ \\
Motivación & 50.45 & 0.14 & $373.99^{\star}$ \\
Administración del Tiempo & 43.72 & 0.23 & $187.59^{\star}$ \\
Ansiedad & 11.62 & 0.35 & $32.96^{*}$ \\
Concentración & 54.52 & 0.18 & $298.35^{*}$ \\
Procesamiento de información & 26.93 & 0.17 & $161.35^{*}$ \\
Selección de ideas principales & 52.51 & 0.20 & $259.94^{*}$ \\
Ayudas para el estudio & 38.62 & 0.22 & $172.16^{\star}$ \\
Autoevaluación & 25.55 & 0.20 & $130.20^{*}$ \\
Estrategia para el examen & 49.27 & 0.17 & $294.24^{*}$ \\
\hline
\end{tabular}

* $p<, 001$

G. L. de Cluster $=2$

G.L. de error $=563$

El Cluster 2 está constituido por la mayor cantidad de alumnos (215) y se caracterizan por presentar un perfil con puntajes intermedios en todas las escalas del IEAE, por lo que podrían tipificar a este grupo como de "Estrategias Intermedias".
El tercer Cluster incluye a 171 casos, los cuales se caracterizan por presentar bajos puntajes en todas las escalas del IEAE, por lo cual constituye el grupo de "Estrategias Bajas".

Tabla 24

Promedio de puntajes de las escalas del IEAE según Cluster

\begin{tabular}{lccc}
\hline \multicolumn{1}{c}{ Escalas } & $\begin{array}{c}\text { Cluster 1 } \\
\text { "Estrategias } \\
\text { Elevadas" } \\
\mathrm{N}=180\end{array}$ & $\begin{array}{c}\text { Cluster 2 } \\
\text { "Estrategias } \\
\text { Intermedias" } \\
\mathrm{N}=\mathbf{2 1 5}\end{array}$ & $\begin{array}{c}\text { Cluster 3 } \\
\text { "Estrategias Bajas" }\end{array}$ \\
\hline Actitud & 3.33 & 3.22 & $\mathrm{~N}=171$ \\
Motivación & 3.66 & 3.32 & 2.84 \\
Administración del tiempo & 4.21 & 3.69 & 2.57 \\
Ansiedad & 4.28 & 4.01 & 3.45 \\
Concentración & 4.16 & 3.52 & 3.22 \\
Procesamiento de información & 3.72 & 3.17 & 3.26 \\
Seleccción de ideas principales & 4.15 & 3.82 & 2.99 \\
Ayudas para el estudio & 4.21 & 3.84 & 3.11 \\
Autoevaluación & 3.94 & 3.60 & 3.26 \\
Estrategia para el examen & 3.75 & 3.28 & 3.12 \\
\hline
\end{tabular}




\section{Gráfico 2}

Distribución de los puntajes según Cluster

En lo que respecta al análisis de la tabulación cruzada de los Cluster identificados según el tipo de gestión de la universidad, presentado en la tabla 25 , indica que no existen diferencias estadísticas significativas $\left(X^{2}=3.05\right.$, G. L. $=2, p=.218$ ), por lo que se puede concluir que los clusters se distribuyen de forma similar en los tipos de gestión de la universidad.
En lo que respecta al análisis de la tabulación cruzada de los Cluster identificados según el género, presentado en la tabla 26, indica que no existen diferencias estadísticas significativas $\left(X^{2}=2.38\right.$ G. L. $\left.=2 \mathrm{p}=.304\right)$, por lo que se puede concluir que los Cluster se distribuyen de forma similar en los tipos de gestión de la universidad.

Tabla 25

Distribución de los Cluster según el tipo de gestión de la universidad

\begin{tabular}{lcccc}
\hline Tipo de gestión & $\begin{array}{c}\text { Cluster } 1 \\
\text { "Estrategias } \\
\text { elevadas" }\end{array}$ & $\begin{array}{c}\text { Cluster 2 } \\
\text { "Estrategias } \\
\text { intermedias }\end{array}$ & $\begin{array}{c}\text { Cluster } 3 \\
\text { "Estrategias } \\
\text { bajas" }\end{array}$ & Total \\
\hline Estatal & 78 & 102 & 90 & 270 \\
Particular & 102 & 113 & 81 & $47,7 \%$ \\
Total & 180 & 215 & 171 & 296 \\
& $31,8 \%$ & $38,0 \%$ & $30,2 \%$ & $56 \%$ \\
\hline
\end{tabular}

$X 2=3.05 \quad$ G. L. $=2 \quad p=.218$ 
Tabla 26

Distribución de los Cluster según género

\begin{tabular}{|c|c|c|c|c|}
\hline Género & $\begin{array}{l}\text { Cluster } 1 \\
\text { "Estrategias } \\
\text { elevadas" }\end{array}$ & $\begin{array}{c}\text { Cluster } 2 \\
\text { "Estrategias } \\
\text { intermedias }\end{array}$ & $\begin{array}{c}\text { Cluster } 3 \\
\text { "Estrategias } \\
\text { bajas" }\end{array}$ & Total \\
\hline Varón & 57 & 83 & 65 & 205 \\
\hline Mujer & 123 & 132 & 106 & 361 \\
\hline Total & $\begin{array}{c}180 \\
31,8 \%\end{array}$ & $\begin{array}{c}215 \\
38,0 \%\end{array}$ & $\begin{array}{c}171 \\
30,2 \%\end{array}$ & $\begin{array}{c}566 \\
100,0 \%\end{array}$ \\
\hline
\end{tabular}

$\mathrm{X} 2=2.38$ G. L. $=2 \quad p=.304$

\section{DISCUSIÓN}

Para iniciar la discusión es necesario referirnos al contexto, y por ello, en primer lugar, trataremos del instrumento de investigación. Este proviene del mundo de la psicología educativa y se inscribe en el contexto teórico cognitivo.

El análisis de los resultados del Inventario de Estrategias de Aprendizaje y Estudio (IEAE) indica que presenta validez de constructo, pues el análisis factorial confirmatorio permite corroborar que el modelo del aprendizaje estratégico se encuentra, en forma consistente y adecuada, en los datos obtenidos en la presente investigación, lo cual coincide con los hallazgos alcanzados en los estudios realizados por Weinstein, Schulte y Palmer (1987), Strucchi (1991), Olaussen y Braten (1998) y Weinstein y Palmer (2002).

Los análisis de los ítems y la confiabilidad por consistencia interna de cada una de las escalas ha permitido apreciar que en todos los casos las correlaciones ítem-test corregidas superaron el criterio propuesto por Kline (1986) y los coeficientes Alfa de Cronbach presentaron valores que pueden ser clasificados como adecuados, lo cual está en concordancia con los hallazgos de Weinstein, Schulte y Palmer (1987), de Strucchi (1991), de Olaussen y Braten (1998), de Weinstein y Palmer (2002), de Corral y Alcalá (2002) y de Flowers (2003).

En términos generales, podemos indicar que el IEAE presenta validez de constructo y confiabilidad por consistencia interna, con lo cual se cumplen con los requerimientos psicométricos básicos para este tipo de instrumentos, tal y como lo proponen Martínez Arias, (1985), Marín (1986), Muñiz (1994, 1996) y Anastasi y Urbina (1998).

Los análisis de la bondad de ajuste a la curva normal, realizados a través de la prueba de Kolmogorov-Smirnov (Siegel \& Castellan, 1995) permitieron conocer que los puntajes de las escalas de actitud, motivación, administración 
del tiempo, ansiedad, concentración, procesamiento de información, selección de ideas principales, ayudas para el estudio y estrategia para el examen y los componentes del aprendizaje estratégico, habilidad, volitivo y autorregulado, presentaron distribuciones de puntajes aproximados a la distribución, lo que permitió conocer cómo se distribuyen en la muestra las escalas del IEAE y, por otro lado, aplicar análisis estadísticos más potentes, con la finalidad de obtener resultados más precisos.

Por otro lado, el análisis comparativo de los alumnos que estudian psicología pertenecientes a universidades con gestión particular presentaron un mejor desempeño en las escalas de actitud, motivación y autoevaluación, es decir que en las universidades particulares sus alumnos presentan mayores actividades e interés acerca de la actividad académica, mayor disposición e incentivos para trabajar, diligencia y autodisciplina y un mayor análisis, revisión y preparación para las clases y los exámenes, que los alumnos de las universidades estatales, estas diferencias podrían deberse a la influencia de los aspectos asociados a las diferentes procedimientos de enseñanza implementados en las universidades particulares y que pueden influir en el uso de ciertas estrategias de aprendizaje en sus alumnos.

En lo que concierne a las diferencias por género, se apreció que las alumnas que estudian psicología pre- sentan un mejor desempeño respecto a los varones en las escalas de actitud, administración del tiempo, ansiedad y ayudas para el estudio, lo cual nos indica que las mujeres tienden a presentar mayores actividades e interés acerca de la actividad académica, un mayor análisis de los principios de aprovechamiento del tiempo, planificación y productividad en relación con las tareas académicas, un mayor grado de preocupación por sus resultados académicos y un mayor uso de técnicas de apoyo o materiales que ayuden a aprender y recordar nueva información, lo cual redunda en que en las mujeres también se encuentre un mayor componente autorregulador del aprendizaje estratégico, lo cual desde el punto de vista de Sizoo, Malhotra y Bearson (2003), es producto de las diferencias individuales de los participantes y no a diferencias de género.

Respecto a las diferencias observadas en la ansiedad, debemos indicar que esto coincide con los hallazgos de Sizoo, Malhotra y Bearson (2003), lo cual podría deberse a la situación de competitividad a la que se enfrentan las mujeres en el ámbito universitario.

En lo que se refiere al resultado en el cual se observó que los alumnos que estudian psicología presentaron un mejor desempeño respecto a las mujeres en la escala de procesamiento de la información, es decir que ellos priorizaron el uso de la elaboración verbal e icónica, del control de la comprensión 
y del razonamiento, esto podría deberse más a las diferencias individuales que a características generales de la muestra estudiada.

Al analizar los resultados globales se apreció que las escalas de actitud, motivación, ansiedad, concentración, procesamiento de información, selección de ideas principales, ayudas para el estudio y estrategia para el examen y los componentes del aprendizaje estratégico de habilidad, volitivo y autorregulación, se encontraban desarrolladas de forma adecuada, lo cual nos corrobora que los estudiantes universitarios evaluados alcanzaron adecuados niveles de desarrollo en sus estrategias de aprendizaje y estudio.

El análisis de Cluster permitió identificar la existencia de tres agrupamientos naturales de estudiantes, de acuerdo con su similitud en los puntajes alcanzados en las escalas del IEAE, en primer lugar encontramos el Cluster 1, al que hemos denominado como de Estrategias Elevadas; se caracteriza por lo que podríamos llamar un mayor comportamiento de aprendizaje estratégico, pues alcanza mayores puntajes en todas las escalas, destacando en las de ansiedad (grado de preocupación por el estudio y el desempeño académico), el uso de ayudas para el estudio (grado en que utilizan técnicas o materiales adecuados para ayudarse a estudiar y recordar nueva información, la administración del tiempo (uso de los principios de la administración del tiempo aplicados en tareas académicas), la concentración (habilidad para prestar atención a las tareas académicas) y la selección de ideas principales (habilidad para seleccionar y escoger información clave y precisa).

En el segundo lugar encontramos el Cluster 2, y constituye el grupo al que denominaremos como de Estrategias de Aprendizaje Intermedias; se distingue por obtener en todas las escalas puntajes intermedios, destacando en ansiedad (grado de preocupación por el estudio y el desempeño académico), el uso de ayudas para el estudio (grado en que utilizan técnicas o materiales adecuados para ayudarse a estudiar y recordar nueva información, selección de ideas principales (habilidad para seleccionar y escoger información clave y precisa), la administración del tiempo (uso de los principios de la administración del tiempo aplicados en tareas académicas) y la concentración (habilidad para prestar atención a las tareas académicas).

En el tercer lugar encontramos el Cluster 3, y constituye el grupo al que denominaremos como de Bajas Estrategias de Aprendizaje; se caracterizan por obtener en todas las escalas puntajes menores intermedios, destacando en la administración del tiempo (uso de los principios de la administración del tiempo aplicados en tareas académicas), la concentración (habilidad para prestar atención a las tareas académicas), el uso de ayudas para el estudio 
(grado en que utilizan técnicas o materiales adecuados para ayudarse a estudiar y recordar nueva información), la ansiedad (grado de preocupación de los estudiantes por el estudio y el desempeño académico) y la autoevaluación (actividades de revisión y preparación de clases y pruebas).

Otro hallazgo interesante radica en que esta clasificación no presenta diferencias estadísticas significativas por el tipo de gestión de la universidad de procedencia ni por género; es decir que los sujetos, en independencia de sus características sociodemográficas, tienden a presentar un funcionamiento característico en lo que concierne a sus estrategias de aprendizaje.

Finalmente, los resultados observados nos llevan a pensar, tal como lo indican Nickerson, Perkins y Smith (1994), que las habilidades metacognitivas que implican el manejo de nuestros propios recursos cognitivos y el control de nuestro propio desempeño cognitivo, son necesarias para mejorar el pensamiento, lo cual conlleva a la necesidad que los alumnos hagan uso de las estrategias para el aprendizaje, pues en la medida en que facilitan un aprendizaje autónomo que exige monitoreo y regulación activa de los procesos cognitivos esenciales para planear, ejecutar y resolver problemas, se van a fortalecer los elementos que sirven de base para la consolidación de un aprendizaje efectivo y de calidad.

\section{REFERENCIAS}

Alarcón, R. (1991). Métodos y diseños de investigación del comportamiento. Lima: Universidad Peruana Cayetano Heredia, Fondo Editorial.

Aliaga, T. J. \& Giove, P. (2003). Diferencias en el MSLQ y el LASSI entre alumnos del quinto año de secundaria con autoevaluación alta y baja del rendimiento académico en matemática. Revista de Psicología, 6(2), 223-236.

Anastasi, A. \& Urbina, S. (1998). Test psicológicos. México: Prentice-Hall.

Arbuckle, J. (2004). AMOS 5.0: Programming reference guide. Chicago: Small Waters Corporation.

Aron, A. \& Aron, E. (2001). Estadística para psicología. Buenos Aires: Pearson Education.

Babbie, E. (2000). Fundamentos de la investigación social. México: International Thomson Editores.

Beltrán, J. (1996) Estrategias de aprendizaje. En: J. Beltrán \& C. Genovard (Eds.). Psicología de la instrucción. Variable y procesos básicos. Madrid: Síntesis.

Bereiter, C. \& Scardamalia, M. (1989). Intentinal learning as a goal of instruction. En: Resnick L. B. (Ed.). Knowing. Learning and instruction. Nueva Jersey: LEA.

Biggs, J. B. (1993). What do inventories of students' learning processes really measure? A theoretical review and clarification. British Journal of Educational Psychology, 63, 3-19.

Brown, A. (1987). Metacognition, executive control, self-regulation, and other more mysterious mechanism. En: F. E. Weinert \& R. H. Kluwe (Eds.). Me- 
tacognition, motivation and understanding. Nueva Jersey: LEA.

Byrne, B. M. (1998). Structural equation modeling with LISREL, PRELIS, and SIMPLIS: Basic concepts, applications and programming. Nueva Jersey: Lawrence Erlbaum Associates Publishers.

Byrne, B. M. (2001). Structural equation modeling with AMOS: Basic concepts, applications and programming. Nueva Jersey: Lawrence Erlbaum Associates Publishers.

Cano, C. E. V. (1996). Estrategias metacognitivas y cognitivas en el aprendizaje: Estudio en alumnos de quinto de secundaria de NSE alto y medio alto en Lima metropolitana. Escuela de Graduados. Tesis para optar el grado académico de magíster. Pontificia Universidad Católica del Perú, 1996.

Clark-Carter, D. (2002). Investigación cuantitativa en psicología: Del diseño experimental al reporte de investigación. México: Oxford Press.

Cohen, J. (1988). Statistical power analysis for the behavioral sciences. Hillsdale, NJ: Erlbaum.

Cohen, J. A. (1992). Power Prime. Psychological Bulletin, 112(1), 155-159.

Cohen, R. J. \& Swerdlik, M. E. (2001). Pruebas y evaluación psicológicas, introducción a las pruebas y a la medición. México: McGraw-Hill.

Corral, N. J. \& Alcalá, M. T. (2002). Dimensiones de las estrategias de aprendizaje y estudio. Congreso Latinoamericano de Educación Superior en el Siglo XXI.

D’Ancona, A. C. (2002). Análisis multivariable. Teoría y práctica en la investi- gación social. Madrid: Editorial Síntesis.

Dansereau, D. F. (1985). A Learning strategy research. En: J. V. Segal; S. F. Chipman \& R. Glaser (Eds.). Thinking and learning skills, 1: Relating instruction to research, Hillsdale, $\mathrm{NJ}$ : Erlbaum.

Derry, S. I. \& Murphy, D. A. (1986). Designing systems that train learning ability. Review of Educational Research, 56, 1-39.

Duffy, G. G. \& Roehler, L. R. (1989). Why strategy instructión is so difficult and what we need to do about it. En: C. B. McCormick; G. Miller \& Pressley, M. (Eds.). Cognitive strategy research: From basic research to educational applications. Nueva York: SpringerVerlag.

Duhaheck, A. \& Iacobucci, D. (2004). Alpha's Standard Error (ASE): An accurate and precise confidence interval estimate. Journal of Applied Psychology, 5, 792-808.

Escurra Mayaute, L. M., Delgado Vásquez, A., Sotil Brioso, A., Pequeña Constantino, J., Quezada Murillo, R., Rivas Castro, G., Solís, R. \& Santos, J. (2004). Influencia de las estrategias de aprendizaje y la reflexión activa sobre el rendimiento escolar de los alumnos de quinto año de secundaria de la ciudad de Lima. Revista de Investigación en Psicología, UNMSM-IPPSi. En prensa.

Fan, X. \& Thompson, B. (2001). Confidence intervals about score reliability coefficients, please: An EPM guidelines editorial. Educational and Psychological Measurement, 4, 517-531. 
Flowers, L. (2003). Test-retest reliability of the Learning and Study Strategies Inventori (LASSI). Reading Research and instructions, 43(1), 31-46.

García, L., Orellana, O. \& Canales, Q. (2002). Factores asociados al rendimiento académico en estudiantes de psicología de la UNMSM. Revista de Investigación en Psicología-UNMSMIPPSi, 5(1), 35-52.

Hair, J. F.; Anderson, R. E.; Tatham, R. L. \& Black, W. C. (1999). Análisis multivariante. México: Prentice Hall International.

Haught, P. A.; Hill, L. A.; Walls, R. T. \& Nardi A. H. (1998). Improved Learning and Study Strategies Inventory (LASSI) and academic performance: The impact of feedback on freshmen. Journal of The First-Year Experience, 10(2), 25-40.

Hernández, S. R.; Fernández, C. C. \& Baptista, L. P. (2003). Metodología de la investigación. México: McGraw-Hill.

Johnson, D. E. (2000). Métodos multivariados aplicados al análisis de datos. México: Internacional Thompson Editores.

Jöreskog, K. \& Sörbom, D. (2004). LISREL 8: Structural equation modeling with the SIMPLIS command language. Nueva Jersey: Lawrence Erlbaum Associates Publishers.

Kerlinger, F. \& Lee, H. (2002). Investigación del comportamiento. Métodos de investigación en ciencias sociales. México: McGraw-Hill.

Kirby, J. R. (1977). Cognitive strategies and educational performance. Orlando: Academic Press.
Kline, P. (1986). A handbook of test construction: Introduction to psychometric design. Londres: Methuen \& Co. Ltd.

Kline, P. (1995). The handbook of psychological testing. Londres: Routledge.

Kurtz-Costes, B. E. \& Schneider, W. (1994). Self-concept, attributional beliefs, and school achievement: a longitudinal analysis. Contemporary Educational Psychology, 19, 199-216.

Ledesma, R. (2004). Aplicación de un programa de cálculo de intervalos de confianza para el coeficiente Alfa de Cronbach. Psico-USF, 9(1), 31-37.

Lohr, S. (1999). Muestreo: Diseño y análisis. México: International Thomson Editores.

Manzano, V. (1997). SOTAM: Sistema de Optimización para el Tamaño de la Muestra. V Congreso de Metodología de las CC. Humanas y Sociales. Sevilla.

Manzano, V. (1998). La calidad del muestreo en las investigaciones sociales. Revista Electrónica de Metodología Aplicada, 1, 16-29.

Marín, G. (1986). Consideraciones metodológicas básicas para conducir investigaciones psicológicas en América Latina. Acta Psiquiátrica y Psicológica de América de Latina, 32, 183-192.

Martínez Arias, R. (1985). Psicometría: Teoría de los test psicológicos y educativos. Madrid: Editorial Síntesis.

Mayor, J.; Suengas, A. \& González, J. (1993). Estrategias metacognitivas. Aprender a aprender y aprender a pensar. Madrid: Síntesis.

Moreno, H. A. (1988). Perspectivas psicológicas sobre la conciencia. Madrid: Universidad Autónoma de Madrid. 
Moreno, H. A. (1989). Metaconocimiento y aprendizaje escolar. Cuadernos de Pedagogía, 173, 53-58.

Muñiz, J. (1994). Teoría clásica de los test. Madrid: Pirámide.

Muñiz, J. (1996). Psicometría. Madrid: Universitas.

Nickerson, R.; Perkins, D. \& Smith, E. (1994). Enseñar a pensar. Madrid: Ediciones Paidós.

Nisbet, J. \& Shucksmith, J. (1986). Estrategias de aprendizaje. Madrid: Santillana S. A.

Nunnally, J. \& Bernstein, I. H. (1995). Teoría psicométrica. México: McGrawHill.

Olaussen, B. S. \& Braten, I. (1998). Identifying latent varianbles measured by the Learning and Study Strategies Inventory (LASSI) in Norwegian College Students. The Journal of Experimental Educational, 67(1), 82-96.

Olaussen, B. S. \& Braten, I. (1999). Students' use of strategies for self-regulated learning: Cross culturl perspectives. Scandinavian Journal of Educational Research, 43(4), 409-82-96.

Palincsar, A. S. \& Brown, A. L. (1984). Reciprocal teaching of comprehensionfostering and comprehension-monitoring activities. Cognition and Instruction. 1, 117-175.

Pérez, C. (2000). Técnicas de muestreo estadístico: Teoría, práctica y aplicaciones informáticas. México: Alfaomega Grupo Editor, S. A. de C. V.

Piaget, J. (1975). La equilibración de las estructuras cognitivas. Problema central del desarrollo. Madrid: Siglo XXI.
Pressley, M.; Presley, F.; Elliot-Faust, D. \& Miller, G. (1985). Children's use of cognitive strategies, how to teach strategies, and what to do if they can't be taugt. En: M. Pressley \& Ch. Brainerd (Eds.). Cognitive learning and memory in children. Nueva York: SpringerVerlag.

Quesada, R. (2001). Cómo planear la enseñanza estratégica. México: Limusa.

Rinaudo, M. C. \& Vélez, G. (2000). Estrategias de aprendizaje y enfoque cooperativo. Buenos Aires: Educando Ediciones.

Salkind, N. J. (2000). Métodos de investigación. México: Prentice-Hall Hispanoamericana.

Sánchez, C. H. \& Reyes, M. C. (2002). Metodología y diseños en la investigación científica: Aplicadas a la psicología, educación y ciencias sociales. Lima: Universidad Ricardo Palma.

Siegel, S. \& Castellan, N. (1995). Estadistica no paramétrica: Aplicada a las ciencias de la conducta. México: Editorial Trillas.

Sizoo, S.; Malhotra, N. \& Bearson, J. (2003). A gender-based comparison of the learning strategies of adult bisness students. College Student Journal, 37(1), 103-110.

Strucchi, E. (1991). LASSI: Inventario de Estrategias de Aprendizaje y Estudio. Buenos Aires: Psicoteca Editorial.

Taylor, N. E. (1983). Metacognitive ability: A curriculum priority. Reading Psychology: An International Quarterly, 4, 269-278.

Vermunt, J. (1995). Process-oriented instruction in learning and thinking strate- 
gies. European Journal of Educational Psychology, 10(4), 325-349.

Visauta Vinacua, B. (1986). Técnicas de investigación social. Modelos causales. Barcelona: Editorial Hispano Europea.

Weinstein, C. \& Mayer, R. E. (1986). The teaching of learning strategies. En: Wittrock, M. C. (Ed.). Handbook of research in teaching, 3. ${ }^{\mathrm{a}}$ edición. Nueva York: Macmillan.

Weinstein, C.; Schulte, A. C. \& Palmer, D. R. (1987). Learning and Study Strategies Inventary (LASSI). Clearwater, FL: $\mathrm{H} \& \mathrm{H}$ Publishing.

Weinstein, C. (1987). Fostering learning autonomy through the use of learning strategies. Journal of Reading, 590595.

Weinstein, C.; J. Husman \& D. Dierking (2000). Self regulation interventions with a focus on learning strategies. En: Boekaerts, M.; P. Pintrich \& M. Zeidner (2000). Handbook of Self-regulation. San Diego: Academic Press.

Weinstein, C. E. \& Palmer D. R. (2002). Learning and Study Strategies Inventary (LASSI) User's manual. Clearwater: H \& H Publishing.

Yussen, S. (1985): El rol de la metacognición en las teorías contemporáneas del desarrollo cognitivo. En: D. L. ForrestPresley; G. E. MacKinnon \& F. Weller (Eds.). Metacognición, cognición y performance humana. Orlando: Academic Press.

Zabalza, M. A. (2002). La enseñanza universitaria, el escenario y sus protagonistas. Madrid: Narcea Ediciones.

Zabalza, M. A. (2003). Competencias docentes del profesorado universitario. Madrid: Narcea. 